University of Louisville

ThinkIR: The University of Louisville's Institutional Repository

Faculty Scholarship

$6-2014$

\title{
Milky Way red dwarfs in the BoRG survey : galactic scale-height and the distribution of dwarf stars in WFC3 imaging.
}

Benne W. Holwerda

University of Louisville

M. Trenti

University of Cambridge

W. Clarkson

University of Michigan - Dearborn

K. Sahu

Space Telescope Science Institute

L. Bradley

Space Telescope Science Institute

See next page for additional authors

Follow this and additional works at: https://ir.library.louisville.edu/faculty

Part of the Astrophysics and Astronomy Commons

Original Publication Information

Holwerda, B. W., et al. "Milky Way Red Dwarfs in the BoRG Survey: Galactic Scale-Height and the Distribution of Dwarf Stars in WFC3 Imaging." 2014. The Astrophysical Journal 788(1): 18 pp.

This Article is brought to you for free and open access by ThinkIR: The University of Louisville's Institutional Repository. It has been accepted for inclusion in Faculty Scholarship by an authorized administrator of ThinkIR: The University of Louisville's Institutional Repository. For more information, please contact thinkir@louisville.edu. 


\section{Authors}

Benne W. Holwerda, M. Trenti, W. Clarkson, K. Sahu, L. Bradley, M. Stiavelli, N. Pirzkal, G. De Marchi, M. Andersen, R. J. Bouwens, and R. Ryan 


\title{
MILKY WAY RED DWARFS IN THE BoRG SURVEY; GALACTIC SCALE-HEIGHT AND THE DISTRIBUTION OF DWARF STARS IN WFC3 IMAGING
}

\author{
B. W. Holwerda ${ }^{1,2,7}$, M. Trenti ${ }^{3}$, W. Clarkson ${ }^{4}$, K. Sahu $^{5}$, L. Bradley ${ }^{5}$, M. Stiavellit $^{5}$, N. Pirzkal ${ }^{5}$,

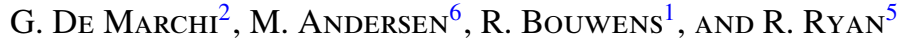 \\ ${ }^{1}$ Leiden Observatory, Leiden University, P.O. Box 9513, 2300 RA Leiden, The Netherlands; holwerda@ strw.leidenuniv.nl \\ ${ }^{2}$ European Space Agency, ESA-ESTEC, Keplerlaan 1, 2200 AG Noordwijk, The Netherlands \\ ${ }^{3}$ Kavli Institute for Cosmology and Institute of Astronomy, University of Cambridge, Madingley Road, Cambridge, CB3 0HA, United Kingdom \\ ${ }^{4}$ Department of Natural Sciences College of Arts, Sciences and Letters, University of Michigan-Dearborn 4901 Evergreen Road, Dearborn, MI 48128, USA \\ ${ }^{5}$ Space Telescope Science Institute, Baltimore, MD 21218, USA \\ ${ }^{6}$ UJF-Grenoble 1/CNRS-INSU, Institut de Planétologie et d'Astrophysique de Grenoble (IPAG) UMR 5274, F-38041 Grenoble, France \\ Received 2013 December 19; accepted 2014 March 11; published 2014 May 23
}

\begin{abstract}
We present a tally of Milky Way late-type dwarf stars in 68 Wide Field Camera 3 (WFC3) pure-parallel fields $\left(227 \mathrm{arcmin}^{2}\right)$ from the Brightest of Reionizing Galaxies survey for high-redshift galaxies. Using spectroscopically identified M-dwarfs in two public surveys, the Cosmic Assembly Near-IR Deep Extragalactic Legacy Survey and the Early Release Science mosaics, we identify a morphological selection criterion using the half-light radius $\left(r_{50}\right)$, a near-infrared $J-H, G-J$ color region where M-dwarfs are found, and a $V-J$ relation with M-dwarf subtype. We apply this morphological selection of stellar objects, color-color selection of M-dwarfs, and optical-near-infrared color subtyping to compile a catalog of $274 \mathrm{M}$-dwarfs belonging to the disk of the Milky Way with a limiting magnitude of $m_{F 125 W}<24(\mathrm{AB})$. Based on the M-dwarf statistics, we conclude that (1) the previously identified north-south discrepancy in M-dwarf numbers persists in our sample; there are more M-dwarfs in the northern fields on average than in southern ones, (2) the Milky Way's single disk scale-height for M-dwarfs is 0.3-4 kpc, depending on subtype, (3) the scale-height depends on M-dwarf subtype with early types (M0-4) high scale-height $\left(z_{0}=3-4 \mathrm{kpc}\right)$ and later types M5 and above in the thin disk $\left(z_{0}=0.3-0.5 \mathrm{kpc}\right)$, (4) a second component is visible in the vertical distribution, with a different, much higher scale-height in the southern fields compared to the northern ones. We report the M-dwarf component of the Sagittarius stream in one of our fields with 11 confirmed M-dwarfs, seven of which are at the stream's distance. In addition to the M-dwarf catalog, we report the discovery of $1 \mathrm{~T}$-dwarfs and $30 \mathrm{~L}$-dwarfs from their near-infrared colors. The dwarf scale-height and the relative low incidence in our fields of L- and T-dwarfs in these fields makes it unlikely that these stars will be interlopers in great numbers in color-selected samples of high-redshift galaxies. The relative ubiquity of M-dwarfs however will make them ideal tracers of Galactic halo substructure with EUCLID and reference stars for James Webb Space Telescope observations.
\end{abstract}

Key words: Galaxy: disk - Galaxy: fundamental parameters - Galaxy: halo - Galaxy: structure - stars: low-mass

Online-only material: color figures, machine-readable table

\section{INTRODUCTION}

Counting stars to infer the shape and size of our Milky Way Galaxy is a classic experiment in astronomy. However, it is also among the most prone to insufficient data as well as conceptual shortfalls (e.g., Herschel 1785; Kapteyn 1922). Over time, it was established that the Milky Way's scale-height is inversely proportional to the masses of stars sampled (Gilmore \& Reid 1983; Gilmore 1984; Siegel et al. 2002). Initially, many of the Galactic models were focused on relatively luminous giant stars and rarely addressed (sub)stellar objects due to completeness issues (see for reviews of star counts and Galactic structure; Bahcall 1986; Gilmore et al. 1989; King et al. 1990; Majewski 1993).

Interest has now shifted to the spatial distribution of (sub)stellar objects, in part because they are possibly the most significant interlopers in studies of extremely high redshift objects (see, e.g., Caballero et al. 2008), but also because these are physically some of the longest-lived objects. Consequently, any deep high-redshift survey, especially in the near-infrared, undertaken with the Hubble Space Telescope (HST) automati- cally becomes a census of the smallest Milky Way stars. More importantly, these objects constitute the most numerous and oldest population of stars in the Galactic disk. For instance, Pirzkal et al. (2005) determined the scale-height of different types of dwarfs from the Hubble Ultra Deep Field (HUDF; Beckwith et al. 2006). Ryan et al. (2005) found L- and T-dwarfs in a small set of Advanced Camera for Surveys (ACS) parallel observations. Stanway et al. (2008) and Pirzkal et al. (2009) determined the Galactic scale-height of M-dwarfs from the Great Observatories Origins Deep Survey (GOODS) fields (Giavalisco et al. 2004). Ryan et al. (2011) added a search of early WFC3 pureparallel fields for L- and T-dwarfs. These studies gradually improved statistics on L-, T-, and M-dwarfs to several dozens of objects.

The identification of these Galactic dwarfs in images is difficult because one needs both high-quality data to distinguish from extragalactic sources and a good understanding of the initial mass function or the local density of these objects as a class. Two of the above studies (Pirzkal et al. 2005, 2009) benefited from spectroscopic identification of the dwarf subtype in the HST/ACS grism spectra of the HUDF and GOODS fields

\footnotetext{
7 Research Fellow.
} 
Table 1

The Scale-heights for the Thin Disk of Dwarf Stars from the Literature

\begin{tabular}{llc}
\hline \hline $\begin{array}{l}\text { Milky Way Scale } \\
\text { Height }\left(z_{0}\right)(\mathrm{pc})\end{array}$ & \multicolumn{1}{c}{ Reference } & Type Stars \\
\hline 300 & Zheng et al. (2001) & \\
$400 \pm 100$ & Pirzkal et al. (2005) & L, T dwarfs \\
$350 \pm 50$ & Ryan et al. (2005) & M4-M9 dwarfs \\
$370 \pm 65$ & Pirzkal et al. (2009) & M0-M9 dwarfs \\
$300 \pm 70$ & Pirzkal et al. (2009) & $>$ M8 \\
$300 \pm 25 \pm 31$ & Ryan et al. (2011) & \\
\hline
\end{tabular}

(the GRAPES ${ }^{8}$ and PEARS ${ }^{9}$ projects, respectively). Combined with an index of stellarity in the direct image, this made identification of the dwarfs nearly certain. Alternatively, Kilic et al. (2005) used high-precision proper motion to identify the Galactic objects. Once an object has been identified as a candidate dwarf, its luminosity distance must be estimated, which requires accurate (sub)typing. Without direct spectra, we will show that this can be readily achieved with sufficient color coverage from the optical to the near-infrared. A dwarf census including subtype and distance can answer several outstanding issues regarding the shape of the Milky Way traced by its most numerous stellar members.

Pirzkal et al. (2009) found, like Stanway et al. (2008), an odd discrepancy in the number of M-dwarfs: there are $24 \%$ more M-dwarfs in the northern than in the southern GOODS field. Because our vantage point in the Milky Way is above the plane of the disk, one would expect the southern GOODS fields to have more M-dwarfs. However, at present, this discrepancy has only been determined from two sight-lines out of the Galaxy, so it is still possible this may be source count field-to-field variance. By studying the older stellar population content of our Galaxy along many sight-lines down to the faintest possible magnitude, we can directly observe and measure the shape of the thin Galactic disk as traced by the old stellar population.

In order to improve our understanding of the contribution of faint dwarfs to Galactic structure, we will need greatly improved statistics to compare against Milky Way structure models. We use the recent $H S T /$ WFC3 pure-parallel searches for bright high-redshift galaxies to find Milky Way dwarfs and determine the parameters of their distribution in the Milky Way disk (Trenti et al. 2011; Yan et al. 2010; Bradley et al. 2012). Thus far, based on HST imaging, the disk scale-height for dwarfs has been found to be $300 \mathrm{pc}$ (Table 1): (Zheng et al. 2001), $400 \pm 100 \mathrm{pc}$ (Pirzkal et al. 2005), $350 \pm 50 \mathrm{pc}$, (L, T dwarfs Ryan et al. 2005), $370 \pm$ $65 \mathrm{pc}$ (for M4-M9 dwarfs), and $300 \pm 70 \mathrm{pc}$ (for M0-M9 dwarfs, Pirzkal et al. 2009) and $300 \pm 25$ ( \pm 31 systematic) pc (>M8, Ryan et al. 2011).

The goal of this paper is to explore the reality of the north-south difference in M-dwarf counts and to accurately determine the scale-height of our Milky Way as traced by M-dwarfs. Previously, Ryan et al. (2011) investigated the L- and T-dwarf content; here we focus our analysis on the M-dwarfs.

The paper is organized as follows: Section 2 reports the properties of the HST/WFC3 observations we use. Section 3 discusses how we generate the relevant SEXTRACTOR catalogs. Section 4 presents our observational calibration of morphological identification of stars, M-dwarfs specifically, and subsequent subtyping of the M-dwarfs. Section 5 presents our catalog of

\footnotetext{
8 Grism ACS Program for Extragalactic Science, see Pirzkal et al. (2004).

9 Probing Evolution And Reionization Spectroscopically, see Pirzkal et al. (2009).
}

Table 2

Summary of the WFC3 Observations of BoRG, CANDELS (v0.5), and ERS on GOODS-S

\begin{tabular}{|c|c|c|c|}
\hline & BoRG & CANDELS & ERS \\
\hline Filters & $\begin{array}{c}F 125 W, \\
F 160 W, \\
F 098 M, \\
F 606 W(F 600 L)\end{array}$ & $\begin{array}{l}F 125 W \\
F 160 W\end{array}$ & $\begin{array}{l}F 125 W \\
F 160 W \\
F 098 M\end{array}$ \\
\hline Pixelscale & 0.08 & $\begin{array}{c}0^{\prime} .06 \\
\text { (resampled to } 0.08 \text { ) }\end{array}$ & 0.08 \\
\hline $\begin{array}{l}\text { Point Spread Function (PSF) } \\
\text { Exposure times } \\
\text { (s) }\end{array}$ & $0^{\prime \prime} 12$ & 0.12 & 0.12 \\
\hline$F 125 W$ & $700-5100$ & 1000 & 5017.61 \\
\hline F160W & $500-3900$ & 1050 & 5017.61 \\
\hline
\end{tabular}

dwarfs in the Brightest of Reionizing Galaxies (BoRG) fields and briefly discusses the validity of our morphological selection. In Section 6 we discuss the north-south difference, the Galactic scale-height as a function of dwarf subtype. Section 7 lists our conclusions and outlines future work.

\section{WFC3 OBSERVATIONS}

We use three WFC3 data sets for this project: the Cosmic Assembly Near-IR Deep Extragalactic Legacy Survey (CANDELS) public v0.5 data release and the Early Release Science (ERS) mosaic, both in the GOODS-South (GOODS-S) field for calibration and characterization of known M-dwarfs, and the BoRG pure-parallel observations for the detection of new Galactic M-dwarfs.

\subsection{CANDELS GOODS-S Observations}

The CANDELS (Grogin et al. 2011; Koekemoer et al. 2011) is designed to explore galaxy evolution, from redshift $z=1.5$ to $\sim 8$. The survey is designed to cover approximately $800 \mathrm{arcmin}^{2}$ and is divided into two parts: the CANDELS/Deep survey on both GOODS-N and GOODS-S and CANDELS/ Wide covering GOODS as well as the Extended Groth Strip (EGS), COSMOS, and UltraDeep Survey (UDS) fields. Data from the survey are nonproprietary and are published in a series of data releases as soon as reduced to scientific quality (see http://candels.ucolick.org). In this paper we use the v0.5 data release of the GOODS-South field, the first epoch of the CANDELS/Deep survey. Exposure times and sensitivity are very similar for this initial mosaic to the BoRG pure-parallel observations (Table 2) in the two near-infrared channels ( $F 125 \mathrm{~W}$ and $F 160 \mathrm{~W}$ ). We resampled the CANDELS $F 125 \mathrm{~W}$ and $F 160 \mathrm{~W}$ mosaics to a pixel scale of $0^{\prime \prime} 08$ using DRIZZLE (Fruchter \& Hook 2002) to conform to the BoRG standard. For subsequent analysis, we only use a subsection of this field (Figure 1), where PEARS and CANDELS coverage overlap.

\subsection{Early Release Science WFC3 Mosaic in Goods-South}

The WFC3 imaging of the GOODS-S field is from the ERS (Windhorst et al. 2011) (see the footprint information at http://candels.ucolick.org/survey/tile_maps/GOODS-S.html and Figure 1). The 10 WFC3 fields in the ERS program were also imaged with the F125W, F160W and F098M filters, the reason it was included in the Ryan et al. (2011) sample. We retrieved the data from the Hubble Legacy Archive (www.hla.stsci.edu) and combined these into a mosaic using SWARP (http://www.astromatic.net/software/swarp) to the 


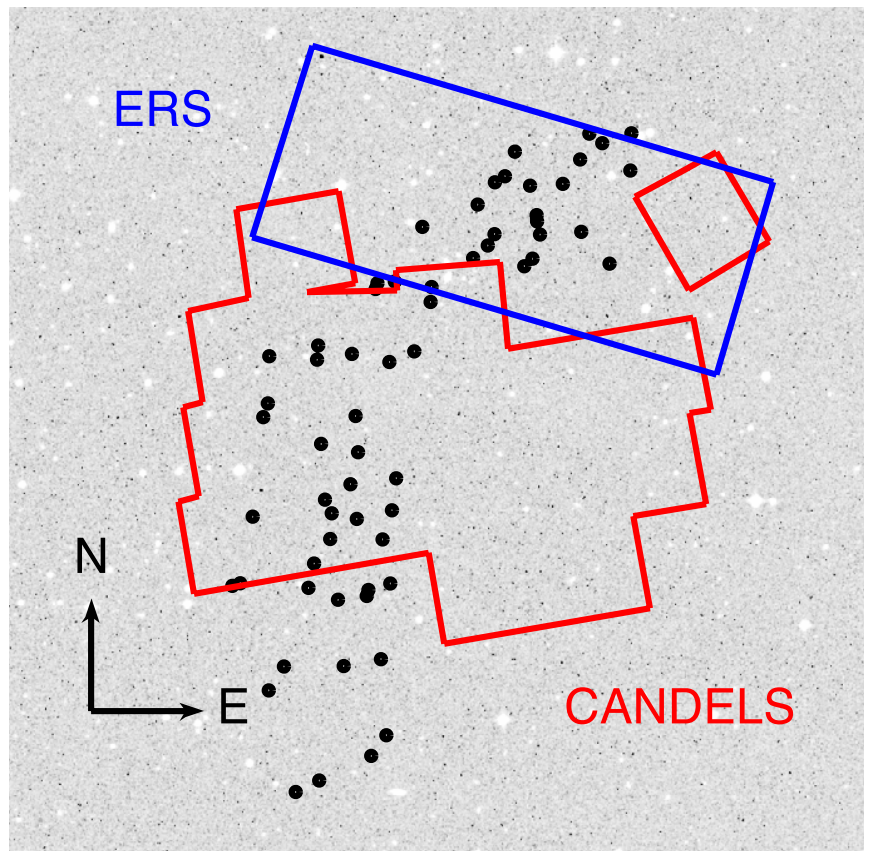

Figure 1. GOODS-South field with the CANDELS v0.5 data release of the deep part of the survey (red) and the Early Release Science mosaics (blue) overlaid on a digital sky survey image. The black circles mark the PEARS-identified stars.

(A color version of this figure is available in the online journal.)

same pixel scale as the BoRG fields. Figure 1 shows the overlap of this mosaic with the PEARS-S survey (Pirzkal et al. 2009).

\subsection{BoRG}

Our principal data set is the WFC3 data from the BoRG (HST GO/PAR-11700; Trenti et al. 2011; Bradley et al. 2012) survey to identify Milky Way dwarf stars from their morphology and color. The BoRG observations are undithered HST/WFC3 conducted in pure-parallel with the telescope pointing to a primary spectroscopic target with the Cosmic Origin Spectrograph (COS; typically a high- $z$ QSO at high Galactic latitude). The limitations for such observations are primarily that no dithering strategy can be used (final images are at WFC3 native pixel scale) and total exposure times are dictated by the primary program.

The program's initial aims were to obtain $176 \operatorname{arcmin}^{2}$ of sky in 38 pointings, using four WFC3 filters (F606W, F098M, $F 125 \mathrm{~W}$, and $F 160 \mathrm{~W}$ ). An extension of the BoRG program in Cycle-19 brought more parallel observations, and the full reproduced set of 68 images is presented in Bradley et al. (2012). Because lines of sight are independent and well separated on the sky mostly at high Galactic latitudes $\left(|b|>20^{\circ}\right.$, see Figure 2), the BoRG survey samples the Milky Way disk away from the plane better than single sight-lines (e.g., the GOODS or $\mathrm{H}(\mathrm{U}) \mathrm{DF}$ fields). The BoRG data set used here is the second data release of 68 WFC3 fields (Table 3) for a total of approximately $209.9 \operatorname{arcmin}^{2}$ as described in detail by Trenti et al. (2011) and Bradley et al. (2012), including some reprocessed fields from another pure-parallel program with similar science goals (Hubble Infrared Pure Parallel Imaging Extragalactic Survey, HIPPIES, HST GO/PAR-11702, Yan et al. 2010). We use the WFC3 data products generated by the BoRG team (see for details Bradley et al. 2012). This is a standard multidrizzle

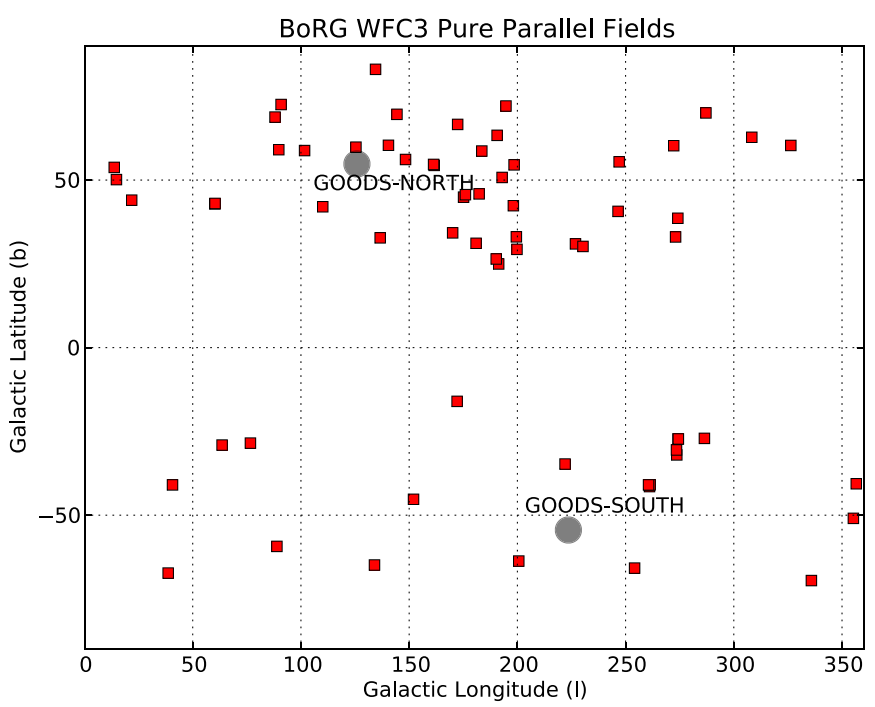

Figure 2. Position of the 68 unique WFC3 pure-parallel fields in Galactic coordinates (red squares). The sampling of the Milky Way disk structure of substellar objects is much better than the two lines of sight by the GOODS/ PEARS fields (black circles). We discard one field (borg_1815-3244) for its low latitudes and its line of sight through the plane of the disk and close to the center of the bulge.

(A color version of this figure is available in the online journal.)

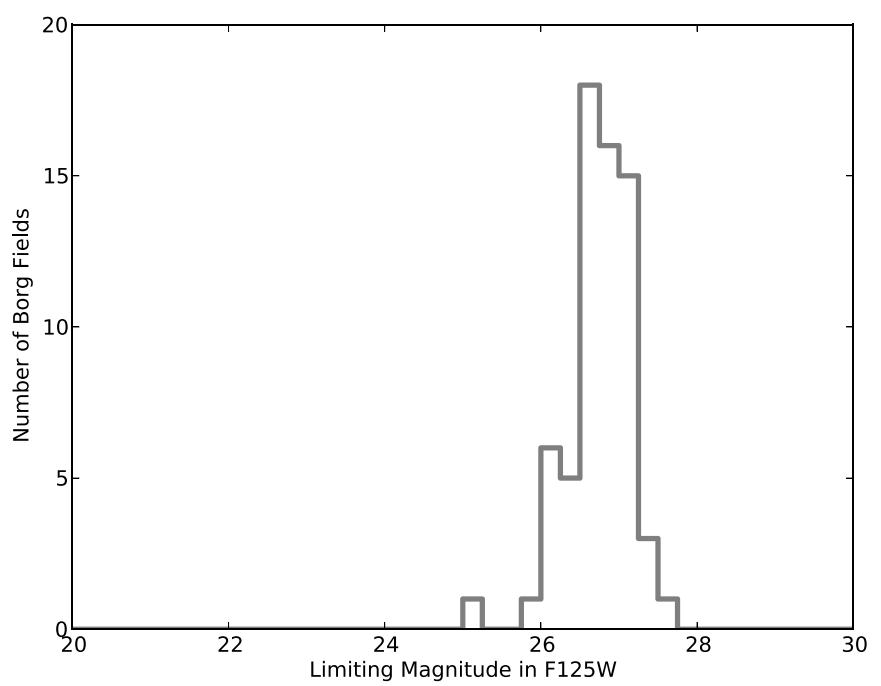

Figure 3. Distribution of limiting magnitude in the $F 125 \mathrm{~W}$ filter from Bradley et al. (2012). Photometric detections are reliable down to $26 \mathrm{mag}$.

reduction of these undithered WFC3 data with Laplacian edge detection (van Dokkum 2001) to the individual flight (FLT) files to mitigate detector hot pixels and cosmic rays. Stellar objects are not affected by this filtering.

The BoRG survey is designed to identify relatively bright $\left(m_{F 125 W} \leqslant 27\right)$ high-redshift galaxies from their broadband colors using the Lyman-break technique (Steidel et al. 1996). The primary aim of the survey is to select redshift $z \sim 7.5$ galaxies as F098M dropouts. Two near-infrared filters ( $F 125 \mathrm{~W}$ and $F 160 \mathrm{~W}$ ) are used for source detection and characterization down to 26 mag (Figure 3). One optical filter $(F 606 \mathrm{~W}$ or $F 600 L P$ in the case of the HIPPIES survey) is used to control contamination from lower redshift sources: $z \sim 1.5$ compact galaxies, active galactic nuclei (AGNs), and cool Milky Way stars. Our interest here now goes to the latter of these interlopers. Given the near-random pointing nature of the pure-parallel HST 
Table 3

Basic Data for the BoRG Fields of the Second Data Release from Bradley et al. (2012) and Schmidt et al. (2014)

\begin{tabular}{|c|c|c|c|c|c|c|c|c|c|c|c|c|c|c|c|}
\hline Field & $\begin{array}{l}\text { Exptime } \\
\text { (s) }\end{array}$ & $\begin{array}{c}\text { Area } \\
\left(\operatorname{arcmin}^{2}\right)\end{array}$ & $\begin{array}{l}\text { R.A. } \\
\text { (J2000) }\end{array}$ & $\begin{array}{c}\text { Decl. } \\
\text { (J2000) }\end{array}$ & $\begin{array}{l}l \\
\left(^{\circ}\right)\end{array}$ & $\begin{array}{l}b \\
\left(^{\circ}\right)\end{array}$ & $\begin{array}{c}z p \\
(\mathrm{mag})\end{array}$ & $\begin{array}{c}z p_{\text {fin }} \\
(\mathrm{mag})\end{array}$ & $\begin{array}{l}\text { Lim } \\
\text { Mag }\end{array}$ & $E(B-V)$ & $A_{F 098 M}$ & $A_{F 125 W}$ & $A_{F 160 W}$ & $A_{F 606 W}$ & $A_{F 600 L P}$ \\
\hline borg_0110-0224 & 13538.2 & 10.9 & 17.531664 & -2.395226 & 133.978373 & -64.865306 & 26.2 & 26.2 & 26.9 & 0.046 & 0.060 & 0.041 & 0.029 & 0.140 & 0.104 \\
\hline borg_0214+1255 & 1402.9 & 3.8 & 33.409984 & 12.915292 & 152.017684 & -45.263536 & 26.2 & 26.1 & 25.9 & 0.122 & 0.158 & 0.109 & 0.077 & $\ldots$ & 0.273 \\
\hline borg_0228-4102 & 1402.9 & 3.7 & 36.986588 & -41.025888 & 254.161019 & -65.793327 & 26.2 & 26.2 & 26.8 & 0.015 & 0.019 & 0.013 & 0.009 & & 0.033 \\
\hline borg_0240-1857 & 1402.9 & 3.6 & 40.114070 & -18.953931 & 200.647911 & -63.687697 & 26.2 & 26.2 & 26.7 & 0.033 & 0.043 & 0.030 & 0.021 & & 0.074 \\
\hline borg_0427+2538 & 702.9 & 4.3 & 66.690208 & 25.640444 & 172.153535 & -16.034094 & 26.2 & 25.4 & 25.1 & 0.906 & 1.173 & 0.809 & 0.574 & & 2.031 \\
\hline borg_0436-5259 & 2905.9 & 3.2 & 69.059214 & -52.986412 & 261.052355 & -41.464289 & 26.2 & 26.2 & 27.3 & 0.007 & 0.009 & 0.006 & 0.004 & & 0.015 \\
\hline borg_0439-5317 & 3005.9 & 3.3 & 69.855420 & -53.278154 & 261.335374 & -40.946634 & 26.2 & 26.2 & 27.2 & 0.005 & 0.006 & 0.004 & 0.003 & & 0.011 \\
\hline borg_0440-5244 & 2002.9 & 3.4 & 69.958786 & -52.731210 & 260.606945 & -40.966425 & 26.2 & 26.2 & 27.0 & 0.006 & 0.008 & 0.006 & 0.004 & & 0.014 \\
\hline borg_0540-6409 & 2308.8 & 1.4 & 84.879133 & -64.150953 & 273.650789 & -32.015266 & 26.2 & 26.2 & 26.6 & 0.057 & 0.074 & 0.051 & 0.036 & 0.172 & \\
\hline borg_0553-6405 & 2611.7 & 1.7 & 88.275723 & -64.088263 & 273.525627 & -30.535388 & 26.2 & 26.2 & 26.8 & 0.045 & 0.059 & 0.040 & 0.029 & 0.137 & \\
\hline borg_0624-6432 & 1205.9 & 3.2 & 95.897657 & -64.528319 & 274.231122 & -27.261687 & 26.2 & 26.2 & 26.4 & 0.058 & 0.075 & 0.052 & 0.037 & 0.174 & \\
\hline borg_0624-6440 & 1205.9 & 3.2 & 95.950899 & -64.662581 & 274.384073 & -27.253871 & 26.2 & 26.2 & 26.6 & 0.059 & 0.076 & 0.053 & 0.037 & 0.178 & $\ldots$ \\
\hline borg_0637-7518 & 2411.7 & 5.0 & 99.265244 & -75.312608 & 286.420817 & -27.079824 & 26.2 & 26.2 & 26.6 & 0.101 & 0.131 & 0.090 & 0.064 & 0.305 & \\
\hline borg_0751+2917 & 5114.7 & 3.5 & 117.709478 & 29.281998 & 191.357531 & 24.959335 & 26.2 & 26.2 & 27.1 & 0.043 & 0.055 & 0.038 & 0.027 & 0.129 & 0.096 \\
\hline borg_0756+3043 & 1905.9 & 3.3 & 118.988521 & 30.717701 & 190.217249 & 26.453514 & 26.2 & 26.2 & 26.6 & 0.062 & 0.081 & 0.056 & 0.040 & 0.189 & \\
\hline borg_0808+3946 & 2205.9 & 3.4 & 122.089304 & 39.758799 & 180.921586 & 31.128951 & 26.2 & 26.2 & 26.6 & 0.045 & 0.059 & 0.040 & 0.029 & 0.137 & \\
\hline borg_0819+4911 & 1205.9 & 3.9 & 124.830368 & 49.183970 & 170.094798 & 34.245407 & 26.2 & 26.2 & 26.5 & 0.051 & 0.066 & 0.046 & 0.032 & 0.155 & \\
\hline borg_0820+2332 & 702.9 & 3.9 & 125.013578 & 23.535374 & 199.823057 & 29.325880 & 26.2 & 26.2 & 26.1 & 0.045 & 0.058 & 0.040 & 0.029 & 0.136 & \\
\hline borg_0835+2456 & 2205.9 & 3.7 & 128.820679 & 24.936409 & 199.532045 & 33.060979 & 26.2 & 26.2 & 26.7 & 0.030 & 0.039 & 0.027 & 0.019 & 0.090 & \\
\hline borg_0846+7654 & 2002.9 & 3.4 & 131.592805 & 76.893333 & 136.609253 & 32.760391 & 26.2 & 26.2 & 27.1 & 0.023 & 0.030 & 0.021 & 0.015 & & 0.052 \\
\hline borg_0906+0255 & 2708.8 & 3.6 & 136.405132 & 2.925182 & 226.847796 & 30.959816 & 26.2 & 26.2 & 27.0 & 0.031 & 0.040 & 0.027 & 0.019 & 0.092 & \\
\hline borg_0909+0002 & 1805.9 & 3.6 & 137.286275 & -0.029911 & 230.317128 & 30.193272 & 26.2 & 26.2 & 26.7 & 0.031 & 0.040 & 0.027 & 0.019 & 0.093 & \\
\hline borg_0914+2822 & 2205.9 & 3.5 & 138.569416 & 28.361907 & 198.147524 & 42.355253 & 26.2 & 26.2 & 26.8 & 0.025 & 0.032 & 0.022 & 0.016 & 0.076 & $\ldots$ \\
\hline borg_0922+4505 & 2105.9 & 3.7 & 140.406384 & 45.087611 & 175.139682 & 44.898070 & 26.2 & 26.2 & 26.5 & 0.020 & 0.026 & 0.018 & 0.013 & 0.060 & \\
\hline borg_0926+4000 & 1105.9 & 4.0 & 141.392945 & 40.005560 & 182.318366 & 45.875115 & 26.2 & 26.2 & 26.2 & 0.015 & 0.020 & 0.014 & 0.010 & 0.046 & \\
\hline borg_0926+4426 & 1602.9 & 3.7 & 141.382123 & 44.426101 & 175.988847 & 45.646771 & 26.2 & 26.2 & 26.7 & 0.016 & 0.020 & 0.014 & 0.010 & & 0.035 \\
\hline borg_1010+3001 & 3211.7 & 3.8 & 152.405996 & 30.018416 & 198.478129 & 54.538030 & 26.2 & 26.2 & 26.8 & 0.024 & 0.032 & 0.022 & 0.015 & 0.074 & \\
\hline borg_1014-0423 & 1105.9 & 3.3 & 153.523466 & -4.378731 & 246.512301 & 40.657070 & 26.2 & 26.2 & 26.2 & 0.036 & 0.046 & 0.032 & 0.023 & & 0.080 \\
\hline borg_1031+3804 & 1505.9 & 3.8 & 157.715302 & 38.058891 & 183.562795 & 58.661992 & 26.2 & 26.2 & 26.3 & 0.014 & 0.018 & 0.012 & 0.009 & 0.041 & $\ldots$ \\
\hline borg_1031+5052 & 4811.7 & 4.4 & 157.690654 & 50.861755 & 161.556783 & 54.378702 & 26.2 & 26.2 & 27.1 & 0.011 & 0.015 & 0.010 & 0.007 & 0.035 & $\ldots$ \\
\hline borg_1033+5051 & 3211.7 & 4.5 & 158.212380 & 50.859570 & 161.288017 & 54.668744 & 26.2 & 26.2 & 26.8 & 0.015 & 0.020 & 0.014 & 0.010 & 0.046 & $\ldots$ \\
\hline borg_1051+3359 & 3317.6 & 3.2 & 162.822134 & 33.985372 & 190.638764 & 63.346845 & 26.2 & 26.2 & 27.0 & 0.026 & 0.033 & 0.023 & 0.016 & 0.078 & $\ldots$ \\
\hline borg_1103-2330 & 4411.7 & 3.5 & 165.807563 & -23.506099 & 273.102916 & 33.023056 & 26.2 & 26.2 & 27.2 & 0.063 & 0.082 & 0.056 & 0.040 & 0.191 & \\
\hline borg_1111+5545 & 2605.9 & 3.2 & 167.736508 & 55.750992 & 148.327174 & 56.140872 & 26.2 & 26.2 & 27.0 & 0.017 & 0.022 & 0.015 & 0.011 & 0.052 & \\
\hline borg_1119+4026 & 1402.9 & 3.7 & 169.513804 & 40.397987 & 172.408816 & 66.608159 & 26.2 & 26.2 & 26.8 & 0.016 & 0.020 & 0.014 & 0.010 & & 0.035 \\
\hline borg_1131+3114 & 1402.9 & 3.7 & 172.876206 & 31.289335 & 194.731674 & 72.096104 & 26.2 & 26.2 & 26.8 & 0.023 & 0.029 & 0.020 & 0.014 & & 0.051 \\
\hline borg_1152+5441 & 2808.8 & 3.5 & 177.958339 & 54.684118 & 140.431973 & 60.372656 & 26.2 & 26.2 & 27.1 & 0.010 & 0.013 & 0.009 & 0.006 & 0.030 & \\
\hline borg_1153+0056 & 2208.8 & 3.6 & 178.182290 & 0.931268 & 272.226679 & 60.255130 & 26.2 & 26.2 & 26.7 & 0.022 & 0.028 & 0.019 & 0.014 & 0.066 & \\
\hline borg_1209+4543 & 3708.8 & 3.6 & 182.354635 & 45.723905 & 144.365550 & 69.616030 & 26.2 & 26.2 & 27.2 & 0.014 & 0.018 & 0.013 & 0.009 & 0.043 & \\
\hline borg_1230+0750 & 1805.9 & 0.5 & 187.469950 & 7.825175 & 287.119671 & 70.032220 & 26.2 & 26.2 & 26.0 & 0.024 & 0.031 & 0.021 & 0.015 & 0.072 & \\
\hline borg_1242+5716 & 2508.8 & 3.5 & 190.553698 & 57.270437 & 125.411526 & 59.813474 & 26.2 & 26.2 & 26.9 & 0.009 & 0.012 & 0.008 & 0.006 & & 0.021 \\
\hline borg_1245+3356 & 1505.9 & 3. & 191.186033 & 33.935834 & 134.469175 & 83.041931 & 26.2 & 26.2 & 26.7 & 0.016 & 0.021 & 0.015 & 0.010 & 0.049 & \\
\hline borg_1301+0000 & 1602.9 & 3.7 & 195.317726 & -0.007321 & 308.309140 & 62.761637 & 26.2 & 26.2 & 26.1 & 0.025 & 0.033 & 0.023 & 0.016 & & 0.057 \\
\hline borg_1337+0028 & 1202.9 & 3.7 & 204.202249 & -0.463529 & 326.341939 & 60.329542 & 26.2 & 26.2 & 26.6 & 0.030 & 0.039 & 0.027 & 0.019 & & 0.067 \\
\hline borg_1341+4123 & 3205.9 & 3.3 & 205.130683 & 41.384472 & 90.818133 & 72.544121 & 26.2 & 26.2 & 27.2 & 0.007 & 0.009 & 0.006 & 0.004 & & 0.015 \\
\hline borg_1408+5503 & 2611.8 & 3.5 & 211.992568 & 55.055640 & 101.682613 & 58.802067 & 26.2 & 26.2 & 26.9 & 0.014 & 0.018 & 0.012 & 0.009 & 0.042 & \\
\hline borg_1437+5043 & 2508.8 & 3.6 & 219.233553 & 50.718933 & 89.759393 & 59.068028 & 26.2 & 26.2 & 27.0 & 0.013 & 0.016 & 0.011 & 0.008 & 0.038 & \\
\hline borg_1524+0954 & 1602.9 & 3.5 & 231.040910 & 9.905686 & 14.750963 & 50.138306 & 26.2 & 26.2 & 26.5 & 0.042 & 0.055 & 0.038 & 0.027 & & 0.095 \\
\hline borg_1555+1108 & 2908.8 & 3.4 & 238.856610 & 11.131677 & 21.791448 & 44.001085 & 26.2 & 26.2 & 27.0 & 0.052 & 0.068 & 0.047 & 0.033 & 0.158 & $\ldots$ \\
\hline borg_1632+3733 & 2405.9 & 3.5 & 248.074403 & 37.557004 & 60.247231 & 42.879109 & 26.2 & 26.2 & 27.0 & 0.010 & 0.014 & 0.009 & 0.007 & 0.032 & \\
\hline borg_1632+3737 & 1205.9 & 3.5 & 247.891785 & 37.608886 & 60.304175 & 43.027091 & 26.2 & 26.2 & 26.6 & 0.010 & 0.013 & 0.009 & 0.006 & 0.029 & \\
\hline borg_2057-4412 & 1202.9 & 3.5 & 4.340154 & -44.206786 & 356.583873 & -40.623775 & 26.2 & 26.2 & 26.4 & 0.038 & 0.049 & 0.034 & 0.024 & $\cdots$ & 0.085 \\
\hline borg_2132+1004 & 1005.9 & 3.8 & 323.061958 & 10.063711 & 63.564901 & -29.075332 & 26.2 & 26.2 & 26.4 & 0.040 & 0.052 & 0.036 & 0.026 & & 0.090 \\
\hline borg_2155-4411 & 1402.9 & 3.7 & 328.812024 & -44.176682 & 355.248970 & -50.945449 & 26.2 & 26.2 & 26.7 & 0.013 & 0.016 & 0.011 & 0.008 & $\cdots$ & 0.028 \\
\hline borg_2203+1851 & 2005.9 & 3.4 & 330.704916 & 18.850050 & 76.655821 & -28.494164 & 26.2 & 26.2 & 26.8 & 0.069 & 0.090 & 0.062 & 0.044 & $\ldots$ & 0.155 \\
\hline borg_2345+0054 & 1402.9 & 3.8 & 356.260909 & -0.901806 & 88.898069 & -59.313470 & 26.2 & 26.2 & 26.8 & 0.036 & 0.047 & 0.033 & 0.023 & & 0.082 \\
\hline borg_2351-4332 & 4208.8 & 3.1 & 357.650020 & -43.525127 & 335.847122 & -69.508926 & 26.2 & 26.2 & 27.3 & 0.013 & 0.017 & 0.011 & 0.008 & & 0.029 \\
\hline borg_0456-2203 & 1809.0 & 3.1 & 73.964600 & -22.048900 & 222.025278 & -34.734134 & 26.2 & 26.3 & 26.7 & 0.038 & 0.037 & 0.028 & 0.020 & 0.095 & $\ldots$ \\
\hline borg_0951+3304 & 2212.0 & 1.8 & 147.700300 & 33.073700 & 192.933397 & 50.779624 & 26.2 & 26.3 & 26.5 & 0.013 & 0.013 & 0.010 & 0.007 & 0.033 & $\cdots$ \\
\hline borg_1059+0519 & 1806.0 & 2.1 & 164.703900 & 5.312500 & 247.014633 & 55.439033 & 26.2 & 26.3 & 26.8 & 0.028 & 0.027 & 0.020 & 0.014 & 0.070 & \\
\hline borg_1118-1858 & 6276.0 & 2.0 & 169.410100 & -18.972600 & 274.146911 & 38.600114 & 26.2 & 26.3 & 27.2 & 0.050 & 0.048 & 0.036 & 0.026 & 0.124 & \\
\hline borg_1358+4334 & 3812.0 & 2.7 & 209.463600 & 43.561000 & 88.017132 & 68.790121 & 26.2 & 26.3 & 27.3 & 0.007 & 0.006 & 0.005 & 0.003 & 0.016 & \\
\hline borg_1459+7146 & 2812.0 & 2.8 & 224.750100 & 71.763800 & 110.071478 & 42.032640 & 26.2 & 26.3 & 27.0 & 0.027 & 0.026 & 0.019 & 0.014 & 0.067 & $\cdots$ \\
\hline borg_1510+1115 & 9529.0 & 1.8 & 227.537100 & 11.241500 & 13.735263 & 53.779237 & 26.2 & 26.3 & 27.7 & 0.046 & 0.045 & 0.033 & 0.024 & 0.115 & \\
\hline borg_2132-1202 & 1809.0 & 1.2 & 322.946700 & -12.039700 & 40.659355 & -40.966360 & 26.2 & 26.3 & 26.2 & 0.062 & 0.060 & 0.045 & 0.032 & 0.153 & \\
\hline borg_2313-2243 & 6326.0 & 3.3 & 348.232600 & -22.725200 & 38.654403 & -67.276574 & 26.2 & 26.3 & 27.1 & 0.026 & 0.025 & 0.019 & 0.013 & 0.065 & \\
\hline
\end{tabular}

program (higher Galactic latitude objects are preferred for COS targets), the BoRG fields are minimally affected by field-tofield (cosmic) variance (Trenti \& Stiavelli 2008). Therefore, this catalog is uniquely positioned to set constraints on the number density of unresolved sources, either Milky Way stars or $z \sim 8$ galaxies.

A recent BoRG observing campaign (BoRG13) is presented by Schmidt et al. (2014), which includes approximately 
Table 4

The M-dwarfs Identified by PEARS in the CANDELS Field

\begin{tabular}{|c|c|c|c|c|c|c|c|c|c|}
\hline ID & Type & R.A. & Decl. & $m_{F 435 W}$ & $m_{F 606 W}$ & $m_{F 775 W}$ & $m_{F 850 W}$ & $m_{F 125 W}$ & $m_{F 160 W}$ \\
\hline 48173 & 4.0 & 53.220073 & -27.854046 & 25.22 & 24.96 & 23.73 & 23.15 & 22.61 & 22.63 \\
\hline 53237 & 4.0 & 53.194890 & -27.848121 & 25.19 & 25.45 & 24.14 & 23.49 & 22.93 & 22.88 \\
\hline 58796 & 1.5 & 53.173782 & -27.841699 & 25.70 & 25.04 & 24.17 & 23.85 & 23.36 & 23.29 \\
\hline 58826 & 4.3 & 53.189870 & -27.841487 & 24.76 & 24.34 & 23.04 & 22.39 & 21.98 & 21.82 \\
\hline 63079 & 1.0 & 53.181657 & -27.836074 & 25.49 & 24.44 & 23.76 & 23.47 & 22.99 & 23.00 \\
\hline 63028 & 3.7 & 53.213644 & -27.835281 & 24.29 & 23.97 & 22.78 & 22.15 & 21.58 & 21.59 \\
\hline 63752 & 3.9 & 53.189345 & -27.834509 & 23.80 & 23.43 & 22.37 & 21.74 & 21.16 & 21.12 \\
\hline 63993 & 2.0 & 53.170843 & -27.833788 & 23.81 & 23.13 & 22.27 & 21.91 & 21.56 & 21.46 \\
\hline 66572 & 8.4 & 53.191395 & -27.830774 & 24.60 & 26.71 & 24.80 & 23.58 & 22.47 & 22.53 \\
\hline 69522 & 6.0 & 53.183514 & -27.826627 & 24.89 & 24.60 & 23.41 & 22.64 & 22.02 & 21.91 \\
\hline 70032 & 3.9 & 53.169502 & -27.825087 & 22.88 & 22.52 & 21.40 & 20.77 & 20.34 & 20.25 \\
\hline 74670 & 1.0 & 53.181116 & -27.817942 & 25.79 & 24.96 & 24.20 & 23.97 & 23.44 & 23.31 \\
\hline 74928 & 4.0 & 53.192508 & -27.815594 & 20.55 & 20.06 & 19.10 & 18.51 & 17.93 & 17.92 \\
\hline 79699 & 4.0 & 53.210190 & -27.808231 & 22.75 & 22.38 & 21.29 & 20.66 & 20.08 & 20.02 \\
\hline 80618 & 2.0 & 53.181804 & -27.808089 & 26.31 & 25.72 & 24.88 & 24.48 & 24.08 & 24.06 \\
\hline 82885 & 0.2 & 53.208736 & -27.804499 & 25.77 & 24.76 & 24.13 & 23.83 & 23.57 & 23.45 \\
\hline 91263 & 0.9 & 53.171308 & -27.793465 & 24.35 & 23.43 & 22.76 & 22.49 & 22.27 & 22.09 \\
\hline 92395 & 2.1 & 53.193512 & -27.792703 & 24.44 & 23.83 & 22.93 & 22.54 & 22.06 & 22.08 \\
\hline 93532 & 2.0 & 53.208197 & -27.791671 & 26.19 & 25.55 & 24.52 & 24.07 & 23.52 & 23.50 \\
\hline 93841 & 4.9 & 53.182786 & -27.791200 & 25.10 & 25.14 & 23.97 & 23.34 & 22.71 & 22.74 \\
\hline 94206 & 4.3 & 53.163637 & -27.790605 & 24.71 & 24.21 & 23.00 & 22.37 & 22.04 & 21.75 \\
\hline 95752 & 3.8 & 53.193173 & -27.788834 & 23.42 & 23.24 & 22.12 & 21.53 & 20.93 & 20.93 \\
\hline 104030 & 0.0 & 53.158515 & -27.777252 & 22.95 & 21.97 & 21.34 & 21.16 & 20.87 & 20.69 \\
\hline 104673 & 0.0 & 53.158184 & -27.773143 & 19.76 & 18.90 & 18.48 & 18.04 & 17.69 & 17.48 \\
\hline
\end{tabular}

Notes. The optical magnitudes are from Pirzkal et al. (2009) and the $F 125 \mathrm{~W}$ and $F 160 \mathrm{~W}$ magnitudes from our SEXTRACTOR catalog of the CANDELS field.

$50 \operatorname{arcmin}^{2}$ of new data (13 fields) and deeper observations of two previous BoRG pointings. This brings the total $J$-band area of the BoRG survey to $\sim 350 \operatorname{arcmin}^{2}$ and makes BoRG the largest existing near-infrared survey with $H S T$, e.g., compared with CANDELS/Wide (260 $\left.\operatorname{arcmin}^{2}\right)$ or Deep (120 $\left.\operatorname{arcmin}^{2}\right)$. We include nine BoRG13 fields as additional lines of sight in our sample (see Table 3).

\section{CATALOG GENERATION}

To construct the catalogs for the BoRG Fields, we ran SEXTRACTOR (Bertin \& Arnouts 1996; Holwerda 2005) with similar settings as Trenti et al. (2011) but set to include MU_MAX, $r_{50}$, and other morphological information. Source detection was done in the $F 125 \mathrm{~W}$ image, with the other filters run in dual-image mode. The multidrizzle weight files were used as rms maps (once normalized, following the prescription in Casertano et al. 2000, WEIGHT_TYPE=MAP_RMS), but scaled appropriately to reflect the noise correlation introduced. The photometric zeropoints are from Dressel et al. (2010); Windhorst et al. (2011): F606W: 26.08, F600LP: 25.85, F098W: 25.68, F125W: 26.25, and $F 160 W: 25.96$, respectively, with $A_{V}$ corrections derived for each individual field (Table 3). These settings resulted in 93106 objects in the BoRG survey.

\section{CANDELS AND ERS CALIBRATION CATALOGS}

We generated SEXTRACTOR v2.8 catalogs with the same settings as above for the section of the resampled CANDELS mosaic, which includes the PEARS-S coverage (Figure 1) to obtain $F 125 \mathrm{~W}$ and $F 160 \mathrm{~W}$ luminosities and morphological information for the spectroscopically identified M-dwarfs. Optical magnitudes for these are from ACS photometry presented in Pirzkal et al. (2009). The overlap between the PEARS-S survey and
CANDELS mosaic is a sample of 24 M-dwarfs (Table 4). We will use their morphology to select bona fide stars in the $F 125 \mathrm{~W}$ images and their colors to select and subtype the M-dwarfs.

A second calibration catalog of 22 bona fide PEARSidentified M-dwarfs is constructed based on the ERS mosaics (Table 5). The ERS mosaic pixel scale was set to be identical to the BoRG fields, and all of the SEXTRACTOR parameters are kept identical to the BoRG ones. Similar to the CANDELS and BoRG catalog, detection was done in $F 125 W$ with the catalogs in F160W, F098M, and F606W or F600LP in dual mode for the respective photometry. Cross-correlation with the PEARSidentified sources used the published right ascension and declination from Pirzkal et al. (2009) converted to pixels, but due to some lingering issues with the WCS coordinates in the ERS data, we are less certain about the cross-identification than in the CANDELS data ( $\sim 0^{\prime \prime} .1$ uncertainty in position). Because of these issues and the uncertainty in the flux calibration between CANDELS and the ERS mosaics (Figure 4), we adopt the CANDELS mosaic as our primary calibrator for morphological selection and subtyping and the ERS to calibrate the color-color selection and as a check of the CANDELS data.

\subsection{CANDELS and ERS Photometry}

We used the same zero-points for all of the filters as those used for the BoRG survey (Trenti et al. 2011; Bradley et al. 2012) and ERS (Windhorst et al. 2011). However, the ERS data was taken when the WFC3 instrument was still cooling down, and hence one could expect slightly different WFC3 performance between the CANDELS and ERS photometry catalog. To check, we identified stars (using the $r_{50}$ criterion below) in the full ERS mosaic and cross-correlated these with the full CANDELS catalog (derived from the 0.06 mosaic). The overlap is 13 point sources. Figure 4 shows the difference SEXTRACTOR magnitudes 

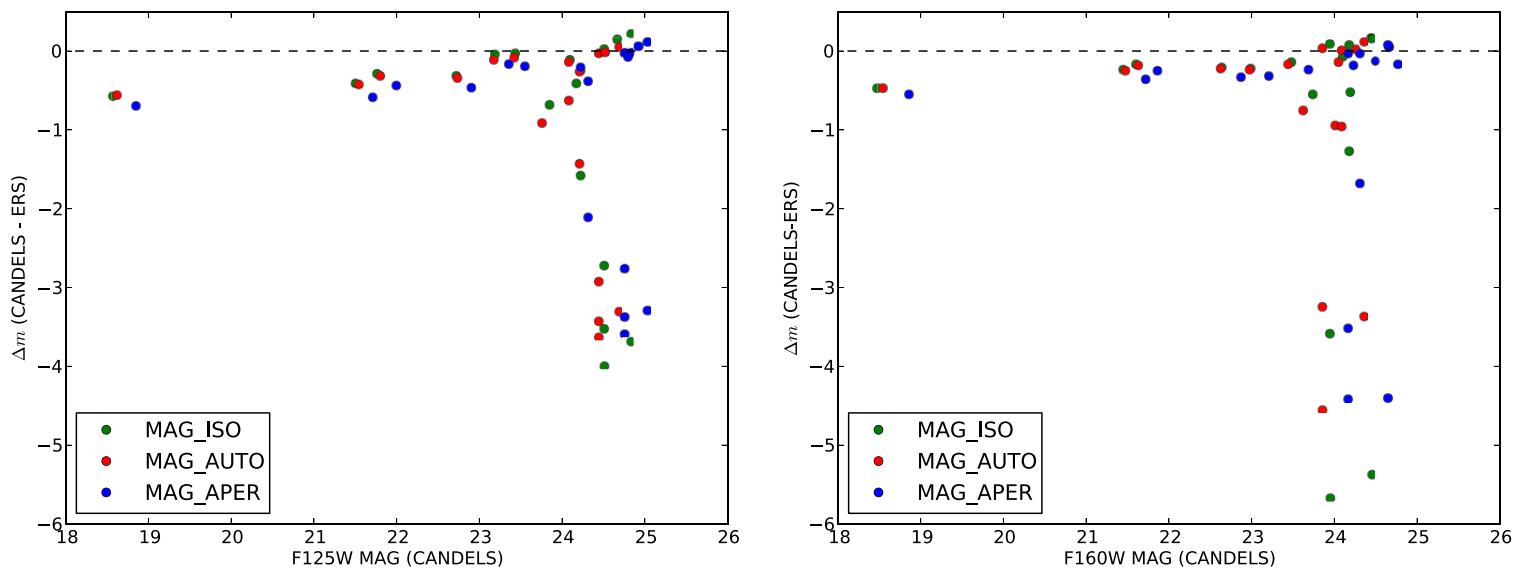

Figure 4. Difference in magnitude between the CANDELS and ERS mosaics. The CANDELS SEXTRACTOR catalog was generated from the 0'.06 pixel scale, full mosaic. Cross-correlation with the ERS mosaic yielded 13 stellar objects. There is an offset in both filters between the mosaics.

(A color version of this figure is available in the online journal.)

Table 5

The M-dwarfs Identified by PEARS in the ERS Mosaic

\begin{tabular}{lccccccccc}
\hline \hline ID & Type & R.A. & Decl. & $m_{F 435 W}$ & $m_{F 606 W}$ & $m_{F 775 W}$ & $m_{F 850 W}$ & $m_{F 125 W}$ & $m_{F 160 W}$ \\
\hline 108497 & 1.7 & 53.093027 & -27.735838 & 21.39 & 20.58 & 19.78 & 19.42 & 19.19 & 19.07 \\
108014 & 2.0 & 53.057966 & -27.735053 & 19.76 & 18.81 & 18.37 & 17.80 & 17.64 & 17.47 \\
110839 & 2.0 & 53.089621 & -27.733082 & 23.02 & 22.36 & 21.48 & 21.06 & 21.52 & 21.30 \\
111269 & 3.0 & 53.113985 & -27.732740 & 24.68 & 23.98 & 23.05 & 22.58 & 21.99 & 22.05 \\
111982 & 6.0 & 53.107958 & -27.728253 & 21.63 & 21.19 & 19.94 & 19.11 & 18.51 & 18.41 \\
114688 & 0.0 & 53.086462 & -27.724381 & 24.06 & 23.11 & 22.49 & 22.22 & 22.73 & 22.41 \\
115223 & 4.0 & 53.105121 & -27.724124 & 25.11 & 24.64 & 23.51 & 22.90 & 22.16 & 22.18 \\
114563 & 6.0 & 53.069511 & -27.723391 & 22.27 & 21.88 & 20.69 & 19.90 & 19.37 & 19.12 \\
116612 & 4.0 & 53.134575 & -27.721317 & 24.55 & 23.85 & 22.70 & 22.24 & 21.63 & 21.53 \\
117391 & 1.3 & 53.087638 & -27.719656 & 23.01 & 22.13 & 21.42 & 21.04 & 20.66 & 20.67 \\
119050 & 2.7 & 53.087816 & -27.717329 & 26.03 & 24.77 & 23.84 & 23.42 & 23.02 & 22.81 \\
120814 & 4.2 & 53.111938 & -27.713369 & 24.19 & 23.99 & 22.87 & 22.19 & 21.70 & 21.61 \\
123686 & 5.3 & 53.090399 & -27.706629 & 23.60 & 23.44 & 22.25 & 21.52 & 20.79 & 20.72 \\
124539 & 8.7 & 53.076943 & -27.706018 & 25.28 & 25.75 & 24.27 & 23.22 & 22.64 & 22.52 \\
124624 & 1.0 & 53.104778 & -27.705292 & 23.78 & 23.41 & 22.76 & 22.42 & 21.25 & 21.08 \\
125478 & 9.0 & 53.100622 & -27.703149 & 24.03 & 24.37 & 22.53 & 21.29 & 20.38 & 20.34 \\
126754 & 1.0 & 53.049225 & -27.701327 & 24.91 & 23.94 & 23.19 & 22.88 & 22.32 & 22.21 \\
128173 & 3.3 & 53.069739 & -27.697205 & 23.69 & 23.12 & 22.11 & 21.57 & 21.11 & 21.09 \\
128247 & 2.0 & 53.096541 & -27.694200 & 21.16 & 20.43 & 19.62 & 19.24 & 18.92 & 18.78 \\
130804 & 1.0 & 53.060606 & -27.691367 & 24.23 & 23.33 & 22.66 & 22.35 & 21.82 & 21.71 \\
132690 & 3.0 & 53.066145 & -27.687565 & 24.21 & 23.86 & 22.91 & 22.44 & 27.13 & 26.77 \\
132690 & 3.0 & 53.065896 & -27.687864 & 24.21 & 23.86 & 22.91 & 22.44 & 22.15 & 22.29 \\
\hline
\end{tabular}

Notes. The optical magnitudes are from Pirzkal et al. (2009) and the $F 125 \mathrm{~W}$ and $F 160 \mathrm{~W}$ magnitudes from our SEXTRACTOR catalog of the ERS mosaic.

Table 6

The Difference and rms between the CANDELS and ERS Magnitudes for the $F 125 W$ and $F 160 W$ Filters

\begin{tabular}{lccc}
\hline \hline Filter & MAG_ISO & MAG_AUTO & MAG_APER \\
\hline F125W & $-0.34(0.23)$ & $-0.40(0.26)$ & $-0.43(0.19)$ \\
F160W & $-0.26(0.11)$ & $-0.28(0.10)$ & $-0.39(0.10)$ \\
\hline
\end{tabular}

Notes. The aperture for MAG_APER is 0!'48 (8-6 pixels, respectively).

for these stars between the CANDELS and ERS mosaics in the $F 125 W$ and $F 160 W$ filters.

Discarding the faintest objects as their stellarity becomes uncertain (Figure 5), the mean difference between the overlap stars for different apertures $\left(m_{F 125 W}<24, m_{F 160 W}<23.5\right)$ are listed in Table 6. We take the differences in the MAG_APER as the most reliable because these were computed for identical-size apertures. Because we used the ASSOC option in SEXTRACTOR, the placement of these apertures could conceivably be slightly different (typically less than a pixel difference), but this is not enough to explain the offset in photometry.

Thus, for the following, we correct the ERS photometry by subtracting 0.43 and 0.39 for the $F 125 W$ and $F 160 W$, respectively. However, we lack similar information for the F098M filter and adopt a correction of $0.4 \mathrm{mag}$, the mean of the correction for the $F 125 \mathrm{~W}$ and $F 160 \mathrm{~W}$ corrections.

\subsection{Morphological Identification of Stars}

To identify the low-mass dwarfs in our catalogs, we opt for a strict morphological selection to allow the color information to be used for identification of the stellar type and subtype. There are several SEXTRACTOR parameter cuts in use to identify unresolved sources, all of them a variation on a concentration 


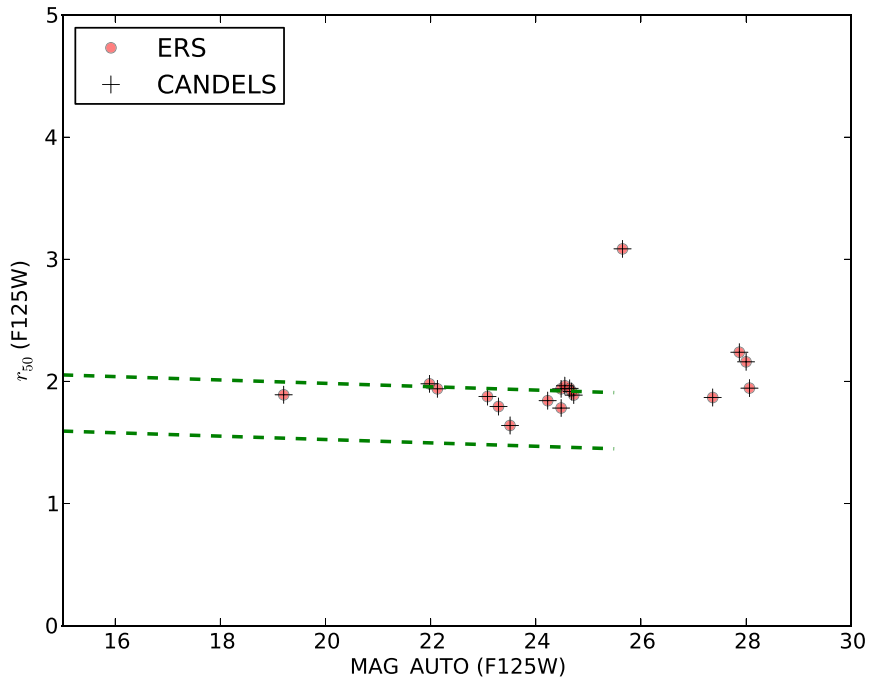

Figure 5. Relation between MAG_AUTO and half-light radius $\left(r_{50}\right)$ for the common objects between the CANDELS v0.5 field and the ERS (see Figure 1). The green lines are the stellarity criterion for the half-light radius. Beyond MAG_AUTO 24, the stellarity of these objects is doubtful.

(A color version of this figure is available in the online journal.)

index; the CLASS_STAR, native to SEXTRACTOR, the half-light or effective radius $\left(r_{50}\right)$ used by Ryan et al. (2011), the flux ratio between to predefined apertures, e.g., the stellarity index $S_{2}=$ $M A G_{\text {aper(1 pix) }} / M A G_{\text {ISO }}(F 125 W)$ ) in Pirzkal et al. (2009), and the relation between the brightest pixel surface brightness and total source luminosity (MU_MAX/MAG_AUTO; Holwerda 2005; Holwerda et al. 2005; Leauthaud et al. 2007, who used it to discard stars).

Using the M-dwarf catalog for CANDELS, we define three morphological criteria: one based on the stellarity index $\left(S_{2}\right)$, one on the MU_MAX/MAG_AUTO ratio, and one for the half-light radius $\left(r_{50}\right)$ for the $F 125 W$ catalog. If we fit the relation between $S_{2}$,MU_MAX/MAG_AUTO, and $r_{50}$ with MAG_AUTO for these M-dwarfs we get

$$
\mid S_{2}+0.0065 \times \text { MAG_AUTO }-1.18 \mid<0.016(0.018),
$$

the relation between MU_MAX and the luminosity (MAG_AUTO),

$$
\mid \frac{\text { MU_MAX }}{\text { MAG_AUTO }}-0.0085 \times \text { MAG_AUTO }-0.65 \mid<0.019
$$

and the half-light radius $\left(r_{50}\right)$,

$$
\mid r_{50}-0.014 \times \text { MAG_AUTO }-2.03 \mid<0.23,
$$

with the variance in the relation determined from the scatter of the M-dwarfs around the fit. Thus, by definition these criteria will typically select all $24 \mathrm{M}$-dwarfs in the CANDELS field. Figures 6, 7, and 8 show these three criteria for objects in the CANDELS subfield catalog with the PEARS M-dwarfs highlighted. For comparison, the ERS M-dwarfs are also marked. Table 7 shows how many stars they select in the CANDELS field.

Our goal is to define criteria that would successfully select the PEARS stars. We note that our calibrators are the confirmed M-dwarfs and that some of those objects selected as stars in the CANDELS field are still Galactic stars, just not M-dwarfs. All three criteria work remarkably better than CLASS_STAR, selecting stars with little contamination for objects brighter than

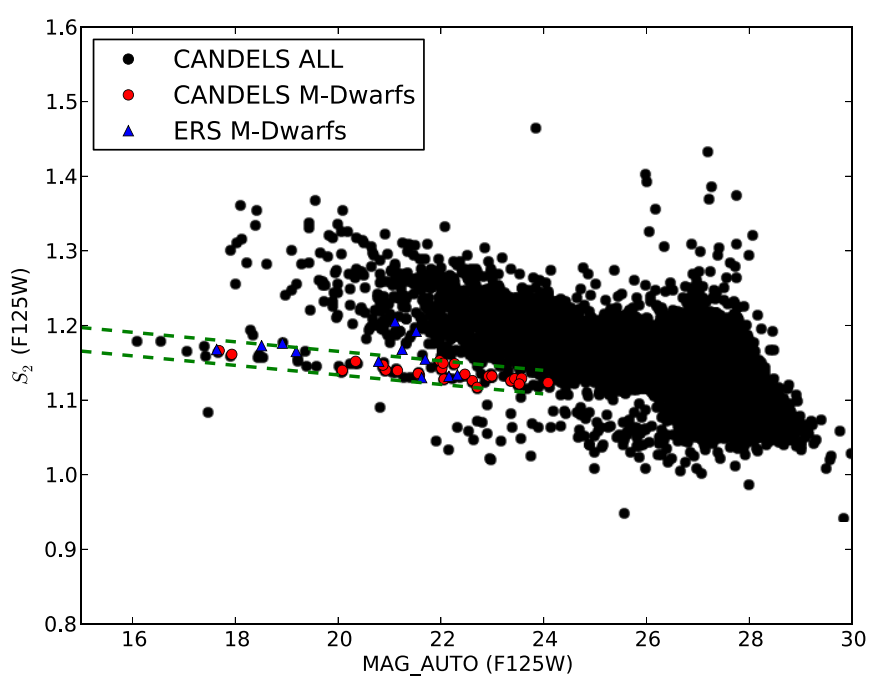

Figure 6. $S_{2}$ parameter for the CANDELS subfield of Figure 1 (crosses) and the bona fide M-dwarfs identified in this field by PEARS (red points). The green dashed lines indicate our $S_{2}$ selection criterion.

(A color version of this figure is available in the online journal.)

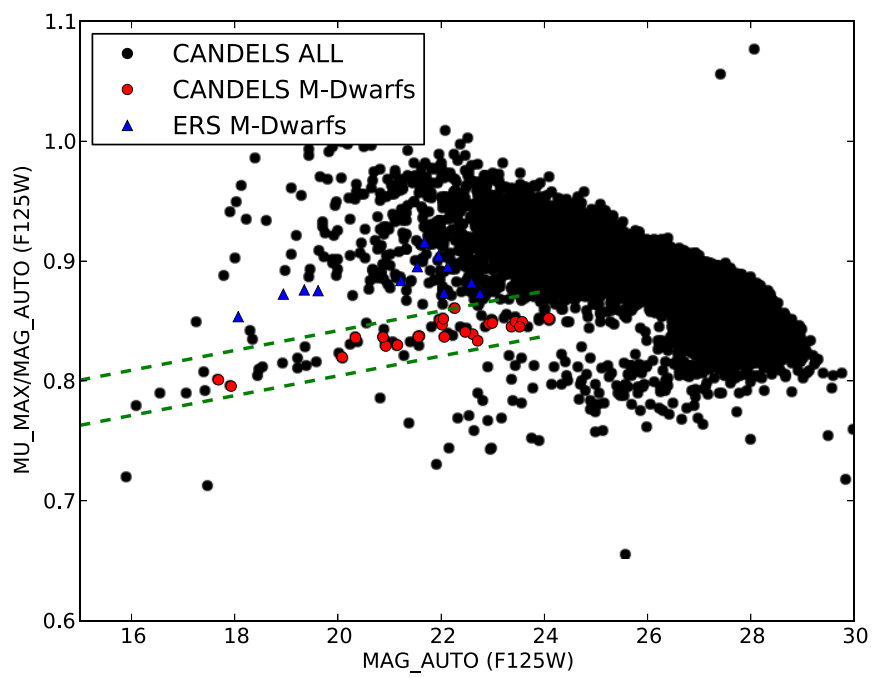

Figure 7. MU_MAX/MAG_AUTO parameter for the CANDELS subfield of Figure 1 (crosses) and the bona fide M-dwarfs identified in this field by PEARS (red circles). The green dashed lines indicate our MU_MAX/MAG_AUTO selection criterion.

(A color version of this figure is available in the online journal.)

Table 7

The Number of Selected Objects in the CANDELS/GOODS-S Mosaic Section (Figure 1) for the Three Different Criteria Separately and in Combinations

\begin{tabular}{lcc}
\hline \hline Criterion & $\begin{array}{c}\text { CANDELS } \\
<25.5\end{array}$ & PEARS M-dwarfs \\
\hline$S_{2}$ & 216 & 24 \\
MU_MAX/MAG_AUTO & 331 & 23 \\
$r_{50}$ & 120 & 23 \\
\hline
\end{tabular}

Notes. The mosaic contains 24 bona fide M-dwarfs and we note their selected number as well. Stricter limits resulted in some of those not being selected.

$m_{F 125 W}<24$ (Figures 6-9). However, there is a marked difference in efficiency between the $S_{2}$ and MU_MAX/MAG_AUTO criteria and the $r_{50}$ criterion in the 24-25.5 mag range. The halflight radius criterion is much stricter than the other two (Table 7), i.e., it includes most of the PEARS-identified M-dwarfs but 


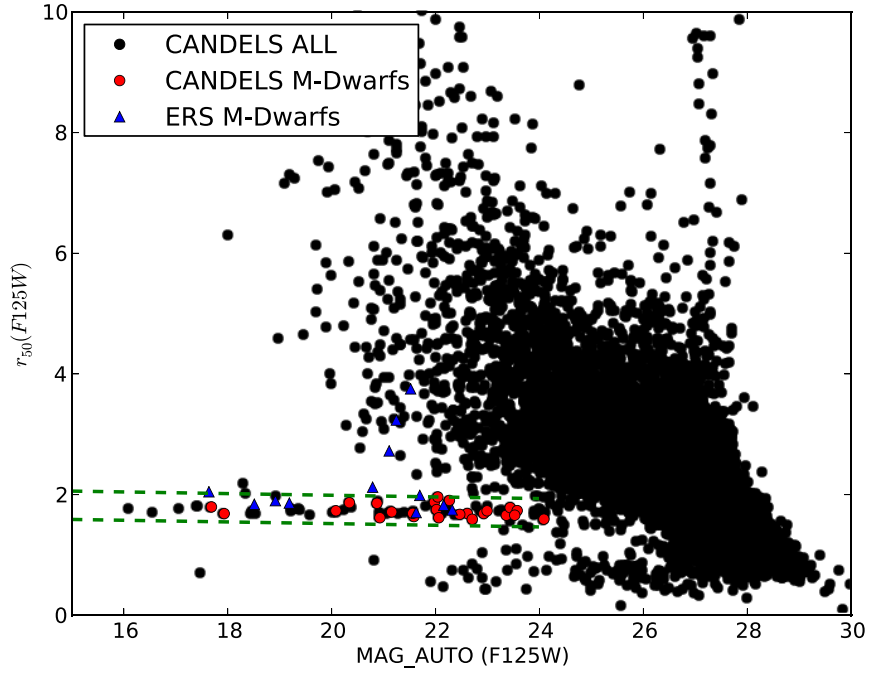

Figure 8. $r_{50}$ parameter (half-light radius) for the CANDELS subfield of Figure 1 (crosses) and the bona fide M-dwarfs identified in this field by PEARS (circles). The green dashed lines indicate our $r_{50}$ selection criterion.

(A color version of this figure is available in the online journal.)

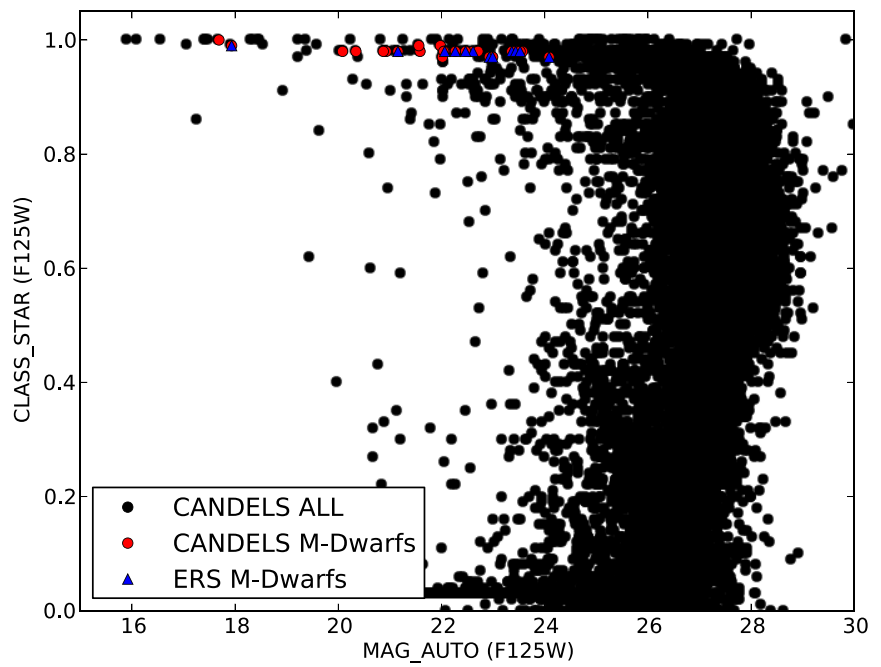

Figure 9. CLASS_STAR parameter from SEXTRACTOR for the CANDELS subfield of Figure 1 (crosses) and the bona fide M-dwarfs identified in this field by PEARS (circles).

(A color version of this figure is available in the online journal.)

includes much fewer objects from CANDELS than the other two criteria.

The half-light parameter was used by Ryan et al. (2011) in some of these fields to select faint dwarf stars (1.2 pixels $\leqslant$ $r_{50} \leqslant 1.8$ pixels with their pixel scale set to $\left.0{ }^{\prime} 09\right)$. Our calibration here shows that this selection criterion is even a little lenient (the selection range can even be narrower), allowing for the different pixel scales, but works very well for the M-dwarfs in these fields.

\subsubsection{ERS Catalog Check}

The 22 M-dwarfs in the ERS catalog are also plotted in Figures 6-9 to serve as a check for the morphological selection. Their positions in these figures reveal a sensitivity in both the $S_{2}$ and the MU_MAX-MAG_AUTO relations for unresolved objects to the pixel scale. The $r_{50}$ criterion is less sensitive to the scale of the pixels. Most likely, this is because it is computed from the growth curve of an object, i.e., the ordered list of pixels.

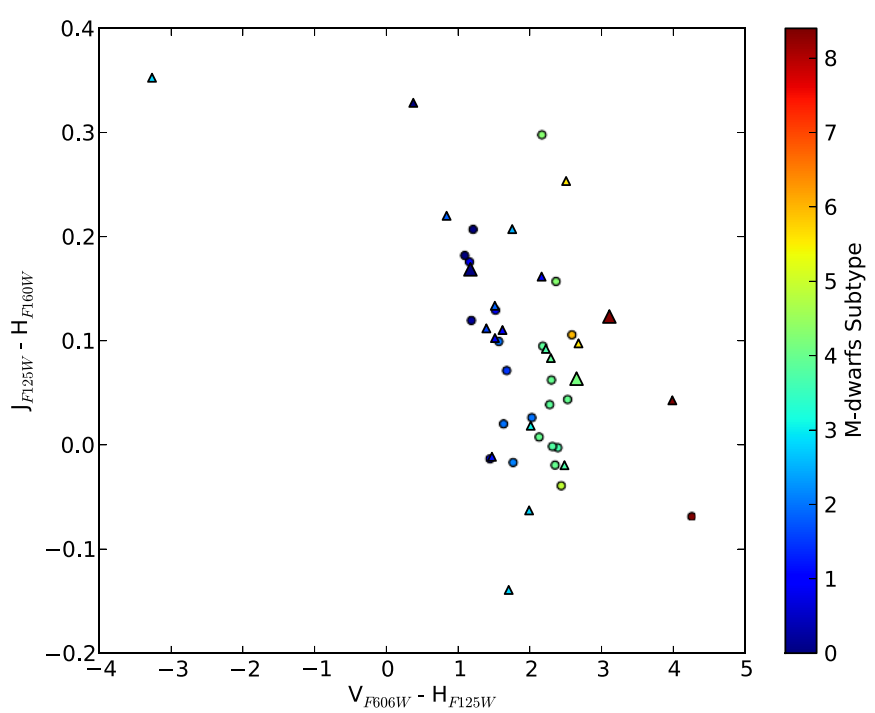

Figure 10. Relation between the $F 606 W-F 125 W$ and $F 125 W-F 160 W$ colors for the M-dwarf subtype (0-8) determined from the GOODS, CANDELS, and ERS data for the PEARS spectroscopically identified M-dwarfs.

(A color version of this figure is available in the online journal.)

The other two criteria both depend on an aperture of a single pixel and hence are more sensitive to the pixel scale. Several of the identified objects in the ERS catalog are well away $\left(r_{50}>2\right.$ pixels $)$ from those values expected for stars, i.e., unresolved objects. These are likely either misidentifications in the ERS field or blends with other objects or artifacts.

The comparison shows that both the $r_{50}$ and the MU_MAX/ MAG_AUTO work well for stellar selection brighter than $24 \mathrm{mag}$, and the $r_{50}$ includes much fewer interlopers down to 25.5 mag. The practical limit of 24 mag for PEARS-identified M-dwarfs in $F 124 \mathrm{~W}$ would limit identifications to distances from 3.2 to $36.3 \mathrm{kpc}$ depending on M-dwarf subtype (M9-M0 respectively), or some 10-100 thin disk scale-heights. We treat our sample as uncontaminated, photometrically accurate, and calibrated with the CANDELS M-dwarfs up to this conservative limit, which is still two magnitudes above the BoRG photometric detection limit (Figure 3).

\subsection{M-dwarf Near-infrared Color-Color Selection}

The second part of the calibration is an empirical color-color selection for the M-dwarfs in the GOODS-S field. We base the M-dwarf selection in near-infrared (NIR) colors because of the wide range in optical-near-infrared colors M-dwarf subtypes can display (Figure 10). To identify M-dwarfs, we require their $J_{F 125 W}-H_{F 160 W}$ and $Y_{F 098 M}-J_{F 125 W}$ color to be similar to the distribution of the PEARS-identified M-dwarfs. To find the $J-H$ color range, we can use both of the CANDELS as the ERS catalog, but for the $Y-J$ color we must rely on the ERS catalog alone.

Figure 11 shows the distribution of $J_{F 125 W}-H_{F 160 W}$ color for the PEARS M-dwarfs in the CANDELS and ERS fields. These are not in the range one would infer from Ryan et al. (2011) in their Figures 3 and 4, but wider by a few tenths of magnitudes. The ERS and CANDELS $J-H$ colors are still different by $\sim 0.1 \mathrm{mag}$ (Figure 11). We suspect that the PEARS M-dwarf $F 125 W-F 160 W$ color distribution in CANDELS in Figure 11 is different than the distribution in Ryan et al. (2011) because their colors were derived from spectra convolved with appropriate filter response functions. There is however an 


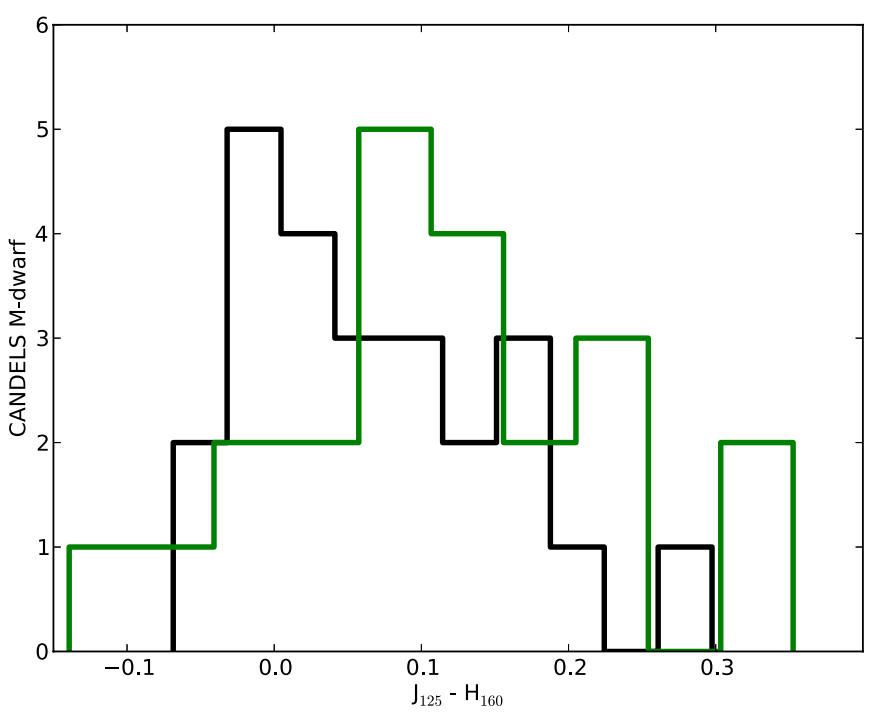

Figure 11. Histogram of the $F 125 W-F 160 W$ colors from CANDELS and ERS of the PEARS spectroscopically identified M-dwarfs.

(A color version of this figure is available in the online journal.)

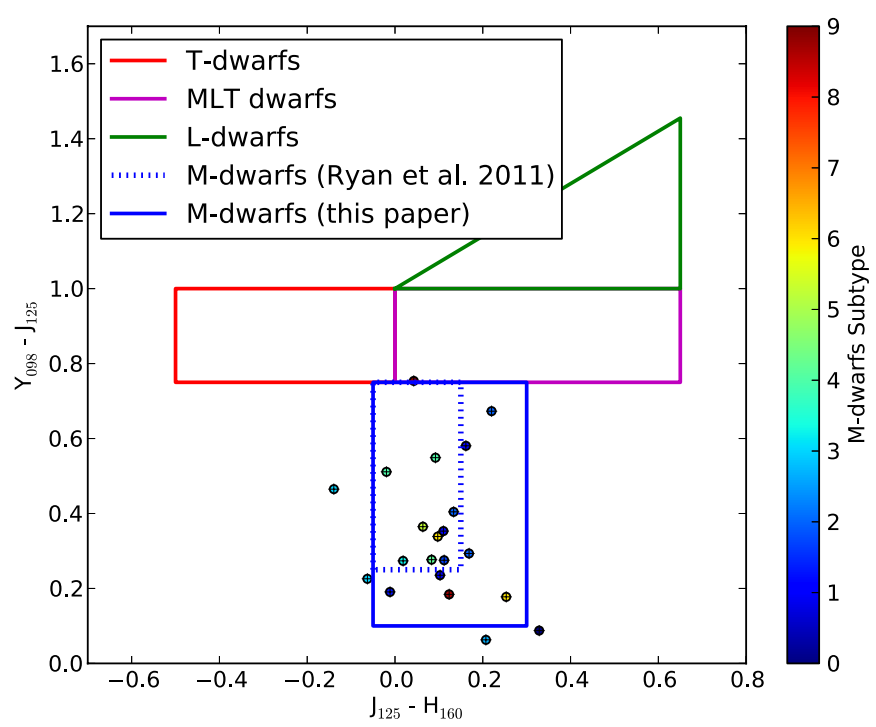

Figure 12. Plot of the $F 125 W-F 160 W$ and $F 098 M-F 125 W$ colors from the ERS of the 22 PEARS spectroscopically identified M-dwarfs.

(A color version of this figure is available in the online journal.)

underreported drop in WFC3 detector sensitivity on the red side of the $F 160 W$ filter (M. Andersen et al., in preparation; M. Andersen et al. 2011, private communication). The F098M and $F 125 W$ filters, however, do not suffer from the detector sensitivity degradation at the red side. This could in part account for the small difference in $J-H$ color of the CANDELS Mdwarfs, whose photometry we trust, with the position of Mdwarfs in Figure 3 in Ryan et al. (2011) or the difference in F125W $-F 160 \mathrm{~W}$ color with the ERS as the detector was still cooling down (Figure 11).

The ERS mosaics are in the same NIR filters as the BoRG fields ( $F 125 \mathrm{~W}, F 160 \mathrm{~W}$, and $F 098 M$ ), and Figure 12 shows the position of the 22 objects matched to PEARS M-dwarfs in a $J-H, Y-J$ color-color plot. We show the same color criteria as Figures 3 and 4 in Ryan et al. (2011). The width and position of the $Y-J$ color criterion is close to what one would infer from Figure 3 in Ryan et al. (2011).

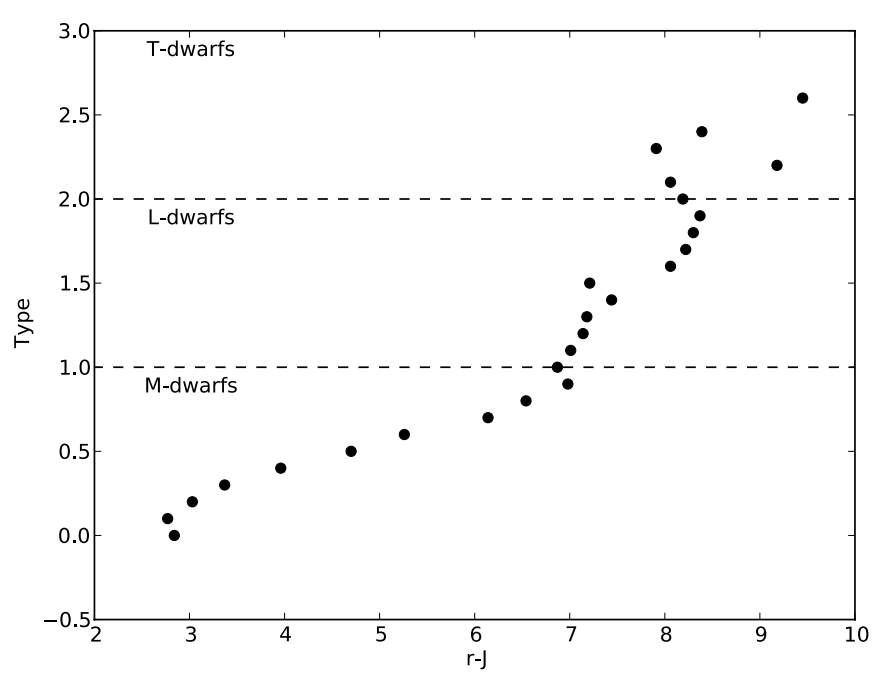

Figure 13. Relation between sdss $-r-J$ color and spectral type from Hawley et al. (2002). M-dwarfs are 0-1 with subtype as the decimal, L-dwarfs are 1-2 with the subtypes as the decimal, and T-dwarfs are type 3 with the decimal the assigned subtype because molecular lines start to dominate the spectrum. The later type dwarfs do not show a good linear relation between a wide optical-nearinfrared color and subtype. However, they do show a redder color than all of the M-dwarf subtypes, and hence we can exclude almost all substellar objects based on color.

Based on both Figures 11 and 12, we define a near-infrared color-color criterion to select M-dwarfs in the BoRG survey; $-0.05<J-H<0.3$ and $0.1<G-J<0.75$ (the solid blue boxes in Figures 12 and 19 in addition to the L, T, and MLT boxes from Ryan et al. (2011)).

\subsection{Subtyping $M$-dwarfs}

Our last calibration involving the PEARS-identified M-dwarfs in the CANDELS and ERS mosaics of the GOODS-S field is to derive a color-subtype relation. Figure 13 shows the relation between $r-J$ color and dwarf type from Hawley et al. (2002). M-dwarfs show a linear relation with a color provided the baseline is long enough, i.e., optical to infrared. The later L- and T-dwarfs do not have such a clear relation.

Figure 14 shows the relation between the $V_{F 606 W}-J_{F 125 W}$ color and the spectroscopic subtype as determined by Pirzkal et al. (2009) for the 24 PEARS M-dwarfs in CANDELS and the 22 PEARS M-dwarfs in the ERS. They obtain a type from template fits to each ACS grism spectrum obtained for these objects. Because of the design of the PEARS observations, there are often several spectra taken at different roll angles. Crosscomparison between the spectral fits show that the uncertainty in the PEARS type classification is less than an M-dwarf subtype. We therefore take these spectral types as our gold standard for the classification of the stellar objects in the WFC3 parallel fields.

Figure 14 shows the relation between the $V-J$ color and spectroscopic type from PEARS. We fit a linear relation to the CANDELS sample because it is the cleanest selection and photometry. The linear relation in the PEARS-identified CANDELS M-dwarfs can be expressed as

$$
M_{\text {type }}=3.39 \times\left[V_{F 606 W}-J_{F 125 W}\right]-3.78 .
$$

The M-dwarfs identified by PEARS in the ERS mosaic display more scatter than the CANDELS M-dwarfs, but after correction of the $F 125 \mathrm{~W}$ magnitudes (see above), the $V-J$ colors and their PEARS subtype generally agree well with the 


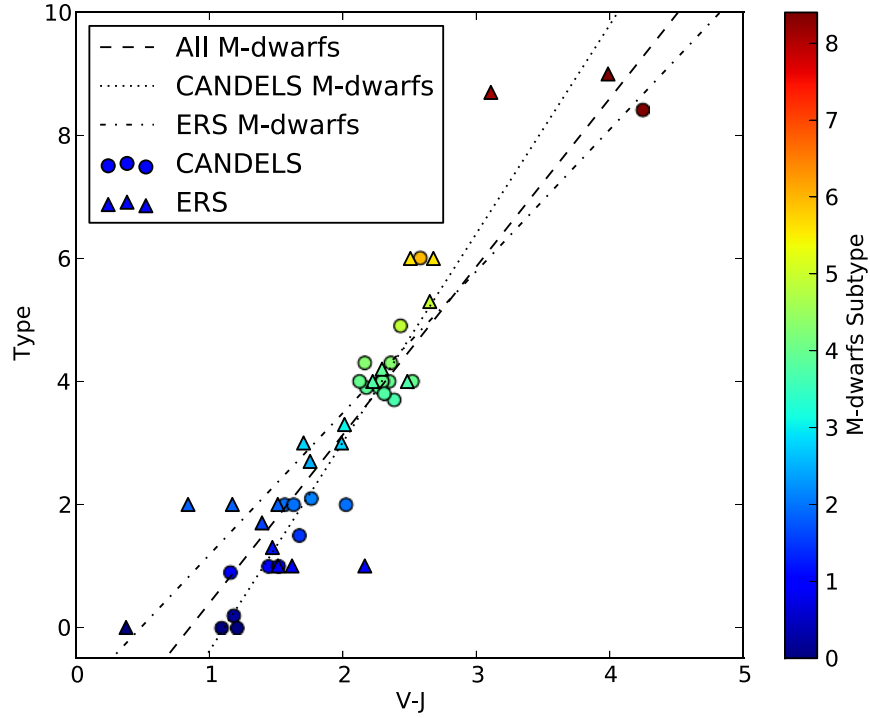

Figure 14. Relation between M-dwarf subtype (0-8) and the F606W-F125W color relation as determined from the GOODS, CANDELS, and ERS data for the PEARS spectroscopically identified M-dwarfs. There is a linear relation between this color and the subtype that we use to type the dwarfs in the BoRG WFC3 parallel fields. We show three fits: one based on all M-dwarfs $(t<$ 6 ), only the CANDELS M-dwarfs $(t<6)$, and only the ERS M-dwarfs. We adopt the CANDELS relation because this sample is the cleanest with accurate photometry and exposure times similar to the BoRG fields.

(A color version of this figure is available in the online journal.)

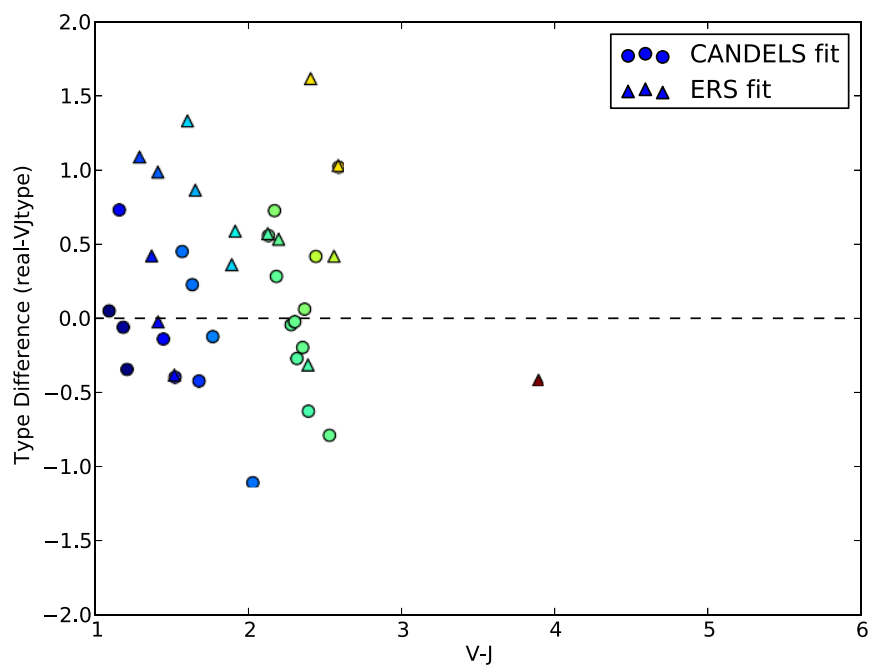

Figure 15. Residual between $V-J$ inferred subtype and the spectroscopic subtype from PEARS for the M-dwarfs in the ERS and CANDELS data. The ERS $F 125 W$ photometry may be shifted slightly still with respect to the CANDELS (Figure 11), and this may account for the higher scatter for this subset.

(A color version of this figure is available in the online journal.)

relation derived from the CANDELS M-dwarfs, although some difference may remain (Figure 11).

Figure 15 shows the residual between the $V-J$ color type and PEARS spectroscopic type. The PEARS M-dwarfs cover mostly subtype $0-5$ and only a few $\mathrm{M}(t>6)$ are in this sample. Consequently, the color-subtype relation is most reliable for the earlier subtypes. Based on Figure 15, we take that our subtype based on $V-J$ color is accurate within a subtype. We adopt this relation to type M-dwarfs in the BoRG fields and compute type uncertainties as a result of the photometric uncertainty in the $F 606 \mathrm{~W}$ and $F 125 \mathrm{~W}$ magnitudes. We use the absolute

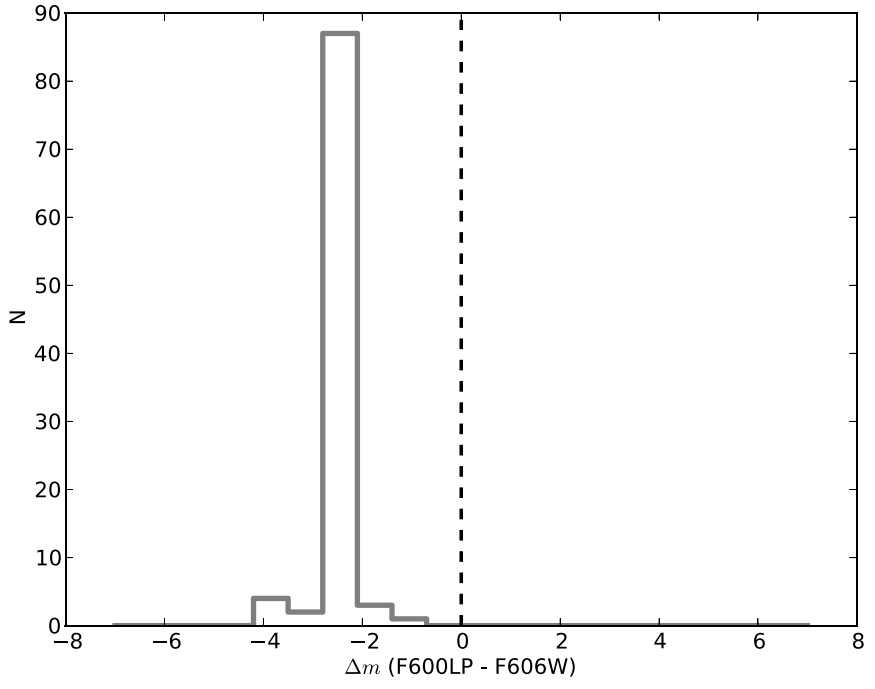

Figure 16. Histogram of the difference in F600W and F600LP MAGAUTO values for those stars in the M-dwarf near-infrared criteria for the three fields in the BoRG survey that have data in both filters.

Table 8

Absolute Two Micron All Sky Survey $J$-band (F125W) Magnitudes of M-dwarfs from Hawley et al. (2002)

\begin{tabular}{lc}
\hline \hline Subtype & $M_{J}$ \\
\hline 0 & 6.45 \\
1 & 6.72 \\
2 & 6.98 \\
3 & 7.24 \\
4 & 8.34 \\
5 & 9.44 \\
6 & 10.18 \\
7 & 10.92 \\
8 & 11.14 \\
9 & 11.43 \\
10 & 11.43 \\
\hline
\end{tabular}

magnitudes from Hawley et al. (2002) to compute distance moduli (Table 8) and infer distances.

\subsection{Substituting F600LP for F606W Photometry}

In the case of 24 fields in the BoRG survey, F606W photometry is not available. These fields come from the HIPPIES or COS-GTO parallel observations, which opted for F600LP rather than F606W. The F600LP filter has a slightly different width compared to the F606W and a different central wavelength (closer to Johnson-I). Fortunately, there are three fields for which both $F 606 W$ and F600LP data are available. We compared the $F 606 W-F 600 L P$ colors for those objects already preselected by their near-infrared color and morphology as likely M-dwarfs to assess the impact of the change of filters. Figure 16 shows the histogram of the color difference between the two bandpasses for M-dwarfs. The difference is substantial: F600L $P-F 606 \mathrm{~W}=$ $-2.65 \mathrm{mag}$ with a spread of $0.88 \mathrm{mag}$. Therefore we correct our F600LP photometry with this difference and increase the uncertainty in the photometry accordingly as well (which should be reflected in the subsequent automated typing).

\section{STARS IN THE BoRG WFC3 PURE-PARALLEL FIELDS}

With the morphological and near-infrared color-color selection in hand, we now search the BoRG fields for M-dwarfs as 


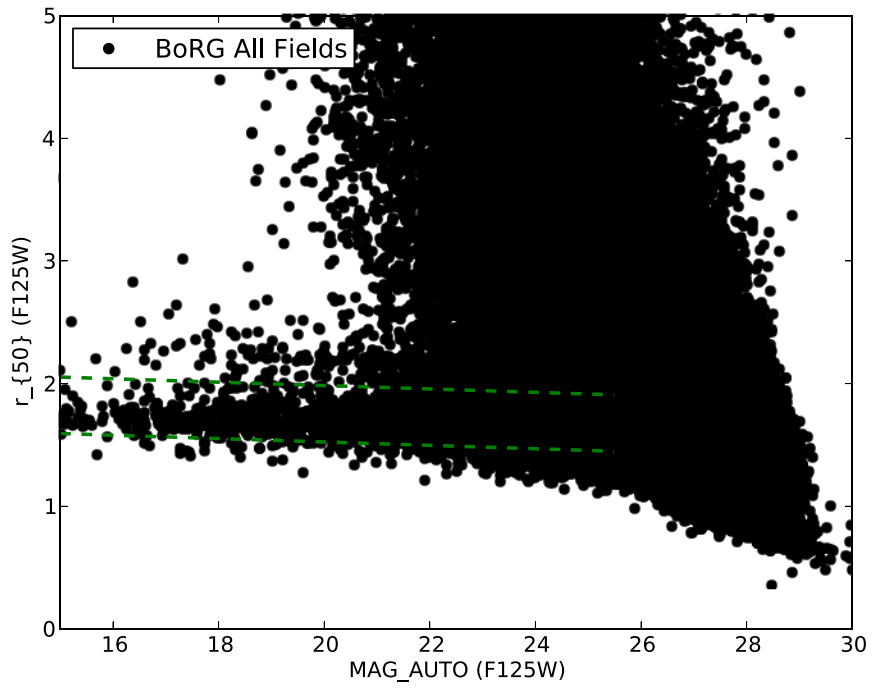

Figure 17. $F 125 \mathrm{~W}$ half-light radius as a function of magnitude for all of the BoRG fields. The green dashed lines are the selection criteria from Equation (3) to identify stars in these fields. Objects below the bottom green line are more likely to be defects (e.g., remaining hot pixels).

(A color version of this figure is available in the online journal.)

well as other substellar types. Figure 17 shows the same parameter space as Figure 8 but with all of the BoRG objects. Based on the $r_{50}$ selection criterion, we find 1308 stars to 24 mag in $F 125 \mathrm{~W}$ in the 59 WFC3 parallel fields. For comparison, Figure 18 shows the other morphology criteria. The $r_{50}$ selection criterion seems the most appropriate for the BoRG fields because the locus of stellar points is within the criterion lines. $S_{2}$ appears equally applicable, but the MU_MAX/MAG_AUTO criterion shows a discrepancy compared to Figure 7. We suspect this is because the CANDELS data was originally at a different pixel size, and this criterion is sensitive to the exact pixel scale of the image. Figure 17 validates therefore our adoption of the $r_{50}$ criterion for the morphological selection of stars.

To identify the various subtypes of stars, we construct an NIR color-color diagram similar to Figure 3 in Ryan et al. (2011), as discussed above. Figure 19 shows this plot with the limits for our selection criteria for approximate types. In the BoRG SEXTRACTOR catalog, we identify 30 L-dwarfs, 1 T-dwarfs, 274 M-dwarfs, and 29 M-, L-, or T-dwarfs based on the color cuts. The M-dwarf sample is the one of interest here.

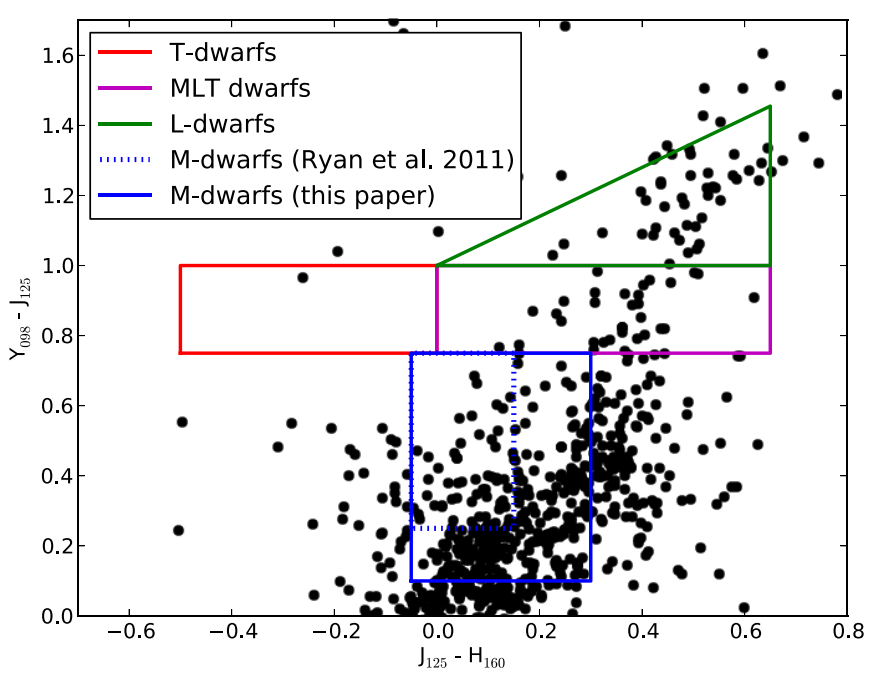

Figure 19. $J_{F 125 W}-H_{F 160 W}$ vs. $G_{F 098 M}-J_{F 125 W}$ color diagram, similar to Figure 4 from Ryan et al. (2011), to distinguish between L-, T-, and M-dwarfs. (A color version of this figure is available in the online journal.)

Tables 14, 15, and 16 list the properties of the identified stars that conform to the M-, T-, and L-dwarf color cuts (Figure 19).

Subsequently, we subtype the M-dwarfs according to Equation (4). Figure 20 shows the histogram of M-dwarf subtypes in the BoRG fields as well as those objects in the MLT box in Figure 19 for comparison. In effect this is an additional optical-near-infrared color criterion for M-dwarf selection because we will discard any star that could not be subtyped. The dominant type in our M-dwarf sample is M0, specifically -1 to 1 type, not unexpectedly because these are the brightest and most numerous type of M-dwarf. There are almost as many later-type M-dwarfs (M4 and above) in the MLT color selection as in our exclusively M-dwarf box. However, as we note above, the later type classification is the most uncertain and in the MLT box susceptible to L- and T-dwarf contamination.

There is still the possibility of contamination by giants, subdwarfs, and AGNs, but we are confident that the morphological selection, luminosity limit, and the color-color restriction select a very clean sample of Milky Way M-dwarfs.

\subsection{Contamination by M-giants or M-type Subdwarfs}

Bochanski et al. (2014) present a catalog of 404 M-giants from the UKIDSS survey DR8 (2400 $\left.\mathrm{deg}^{2}, K<17\right)$, with
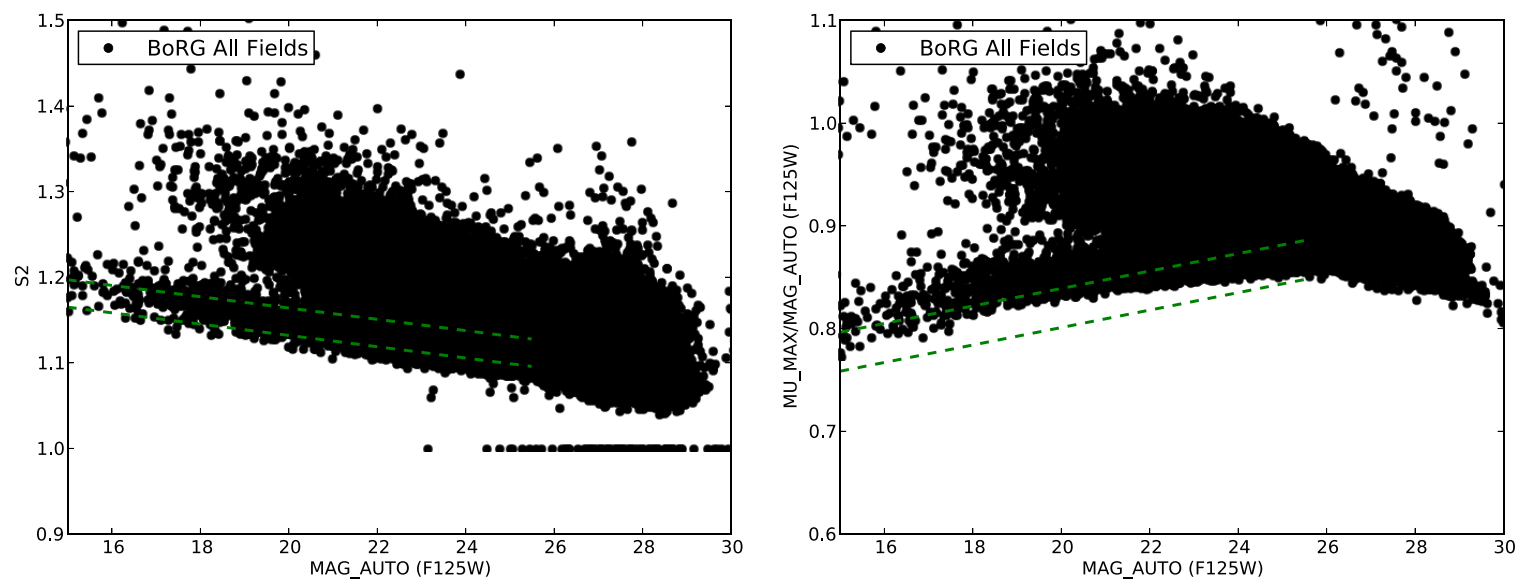

Figure 18. Two alternative morphological selections for stars for the BoRG data: the $S_{2}$ and the mu_max/mag_auto ratio.

(A color version of this figure is available in the online journal.) 


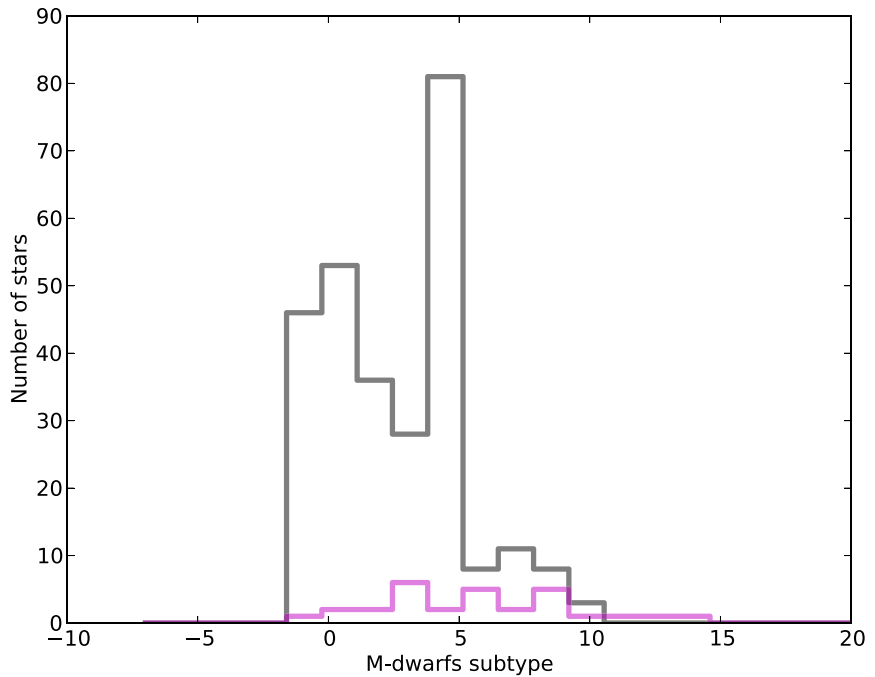

Figure 20. Histogram of M-dwarf types in our BoRG, inferred from Equation (4). M0 and M1 are the most prevalent in the sample. As can be seen in the histogram, some of the stellar objects are not M-dwarfs but either other substellar objects or possibly very distant (intergalactic?) M-giants.

(A color version of this figure is available in the online journal.)

these M-giants among the most distant stars belonging to the Milky Way. In principle, M-giants could be selected by our color selection. However, their on-sky density $(4.3 \times$ $10^{-5} \mathrm{M}$-giants/ $\operatorname{arcmin}^{2}$ ) makes it unlikely not impossible that even a single one is included in our selection. One could make a similar plausibility argument against very nearby M-type subdwarfs: the volume probed at close distances is comparatively small for a pencil beam survey.

\section{DISCUSSION}

Based on our bona fide, morphological- and color-selected M-dwarfs and the subsequent color subtyping, we explore the north-south discrepancy, the Galactic thin disk scale-height as a function of subtype, and whether or not we detect any additional Galactic component (halo or thick disk).

\subsection{The North-South Divide}

Pirzkal et al. (2009) found a north-south discrepancy between the number of M-dwarfs in the PEARS fields. They find 51 and 63 M0-9 dwarfs in the GOODS north and south fields, respectively. Accounting for the different field sizes (41.61 $\mathrm{arcmin}^{2}$ and $59.50 \operatorname{arcmin}^{2}$, respectively), this is equivalent to 1.23 and $1.06 \mathrm{M}$-dwarfs $\operatorname{arcmin}^{-2}$, an excess of $11 \%$ in the northern field. Both GOODS fields are at similar Galactic latitude, and Pirzkal et al. note that because the Sun is north of the Galactic plane, the northern excess appears to be a contradiction. Widrow et al. (2012) and Yanny \& Gardner (2013) find similar north-south discrepancies in the Sloan Digital Sky Survey (SDSS) catalog of (dwarf) stars in the solar neighborhood.

One of our science goals with this study was to establish if this is a real discrepancy due to natural field variance. The BoRG fields are over many more lines of sight and a division of these in Galactic northern and southern fields result in 134.5 and $72.1 \mathrm{arcmin}^{2}$, respectively. We count the M-dwarfs in those fields above $20^{\circ}$ Galactic longitude to avoid two southern fields that sample more along the Galactic plane.

We find on average $1.157 \pm 0.088 \mathrm{M}$-dwarfs of all types (M0-9) per $\operatorname{arcmin}^{2}$ in the northern fields and $1.293 \pm$

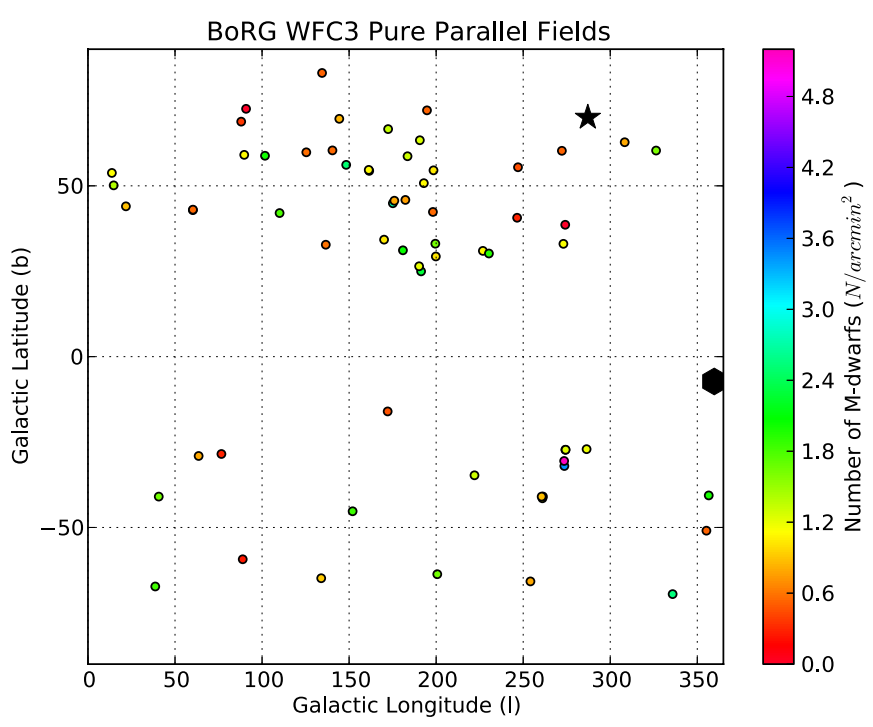

Figure 21. Distribution of BoRG fields with the number of M-dwarfs indicated. One field, borg_1230+0750 (star) stands out with 22 M-dwarfs $\left(\sim 20\right.$ stars $\left.\operatorname{arcmin}^{-2}\right)$. We discard borg_1815-3244 (black circle) for its low latitudes and line of sight through the plane of the disk and close to the center of the bulge.

(A color version of this figure is available in the online journal.)

Table 9

Average Number of M-dwarfs per Square Arcminute in Southern and Northern Galactic Fields $\left(|l|>20^{\circ}\right)$

\begin{tabular}{lcccc}
\hline \hline & $\begin{array}{c}\text { M-dwarfs } \\
\text { North }\end{array}$ & South & $\begin{array}{c}\text { North } \\
\text { /South }\end{array}$ & S/N \\
& & & 0.89 & 8.02 \\
\hline M-dwarfs & $1.157 \pm 0.088$ & $1.293 \pm 0.127$ & 1.10 & 6.45 \\
M0-4 & $0.859 \pm 0.076$ & $0.779 \pm 0.099$ & 0.45 & 4.11 \\
M5-10 & $0.210 \pm 0.038$ & $0.465 \pm 0.076$ & 1.73 & 3.27 \\
M0 & $0.304 \pm 0.045$ & $0.176 \pm 0.047$ & 1.03 & 2.81 \\
M1 & $0.156 \pm 0.032$ & $0.151 \pm 0.043$ & 1.31 & 2.23 \\
M2 & $0.115 \pm 0.028$ & $0.088 \pm 0.033$ & 1.17 & 2.03 \\
M3 & $0.088 \pm 0.024$ & $0.075 \pm 0.031$ & 0.68 & 3.58 \\
M4 & $0.196 \pm 0.036$ & $0.289 \pm 0.060$ & 0.26 & 3.07 \\
M5 & $0.095 \pm 0.025$ & $0.364 \pm 0.068$ & 0.27 & 0.82 \\
M6 & $0.007 \pm 0.007$ & $0.025 \pm 0.018$ & 1.62 & 1.22 \\
M7 & $0.041 \pm 0.017$ & $0.025 \pm 0.018$ & 3.23 & 0.93 \\
M8 & $0.041 \pm 0.017$ & $0.013 \pm 0.013$ & 0.54 & 1.22 \\
M9 & $0.020 \pm 0.012$ & $0.038 \pm 0.022$ & 0.00 & 0.00 \\
M10 & $0.007 \pm 0.007$ & $0.000 \pm 0.000$ & & \\
\hline
\end{tabular}

Notes. M-dwarf numbers refer to those objects in the blue box in Figure 19. The last two columns are ratio of M-dwarfs in northern and southern fields and the signal-to-noise ratio.

$0.127 \mathrm{arcmin}^{-2}$ in the southern Galactic fields (Table 9), which agrees with the previously observed discrepancy. The northern overdensity is the most pronounced in the early (M0-4) type M-dwarfs. Taking into consideration that for these early types the statistics are more secure, and early types dominate the grism sample of Pirzkal et al. (2009) (or the SDSS catalog); our counts corroborate the previously found north-south discrepancy. The northern overdensity is most pronounced in the earliest types, gradually diminishing and reversing for late-type M-dwarfs (Table 9).

\subsection{A Local Overdensity of M-dwarfs}

A single field, borg_1230+0750, stands out from the other BoRG fields with a local density of $21.4 \mathrm{M}$-dwarfs $\operatorname{arcmin}^{-2}$. 


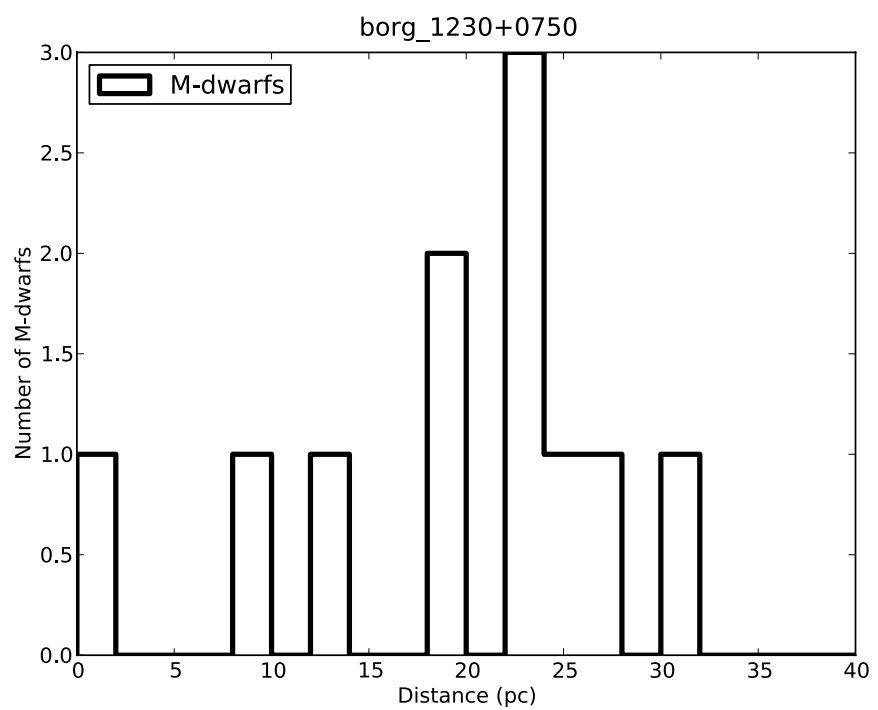

Figure 22. Distribution of M-dwarf distances in borg_1230+0750, with a concentration at $20-25 \mathrm{kpc}$.

Such overdensities of faint Galactic stars are a possible contaminant for identifications of overdensities of high-redshift clusters (e.g., Trenti et al. 2012).

The Galactic position of this overdensity $\left(l=287^{\circ} .119670593\right.$, $b=70.0322203957$, Figure 21) argues against a spiral arm or disk substructure as the origin of this overdensity. However, its position is exactly on the Sagittarius stellar stream (Majewski et al. 2003; Belokurov et al. 2006). Figure 22 shows the histogram of photometric inferred distances for the M-dwarfs in borg_1230+0750. The distance of the overdensity of M-dwarfs at $20-25 \mathrm{kpc}$ is also in line with these M-dwarfs belonging to the Sagittarius stream (Newberg et al. 2002; Belokurov et al. 2014) at this Galactic longitude.

The relative ease with which this stellar stream was identified in the BoRG data shows that the future EUCLID mission will identify virtually all streams in the Milky Way halo from the dwarf stars alone.

\subsection{Thin Disk Scale-Height}

Previous studies based on small numbers of fields have already given us several estimates of the scale-height of the thin disk of the Milky Way in substellar objects (Table 1). The consensus appears to be a scale-height between 300 and $400 \mathrm{pc}$ for the thin disk. For M-dwarfs, Jurić et al. (2008) find a thin disk of $z_{0}=300 \mathrm{pc}$ and a thick disk with $z_{0}=900 \mathrm{pc}$ from SDSS data.

To estimate the distances to each M-dwarf, we compute the distance modulus from the inferred subtype (and hence absolute magnitude) and the apparent magnitude in $F 125 \mathrm{~W}$. We compute the Galactic radius and height above the plane for all M-dwarfs, based on their Galactic longitude, latitude, and the above distance, assuming the position of the Sun is $27 \mathrm{pc}$ above the plane and at a Galactocentric radius of $8.5 \mathrm{kpc}$ from the Galactic center. To compute the density at each M-dwarf's position, we compute the physical area at the inferred distance of the BoRG survey field in which the dwarf was found and a bin-width of $1 \mathrm{pc}$.

We discard one field (borg_1815-3244) for two reasons: it is at low Galactic latitude with a line of sight straight through the Galactic bulge (Figure 21), and there is only F600LP information available, which makes subtyping of M-dwarfs

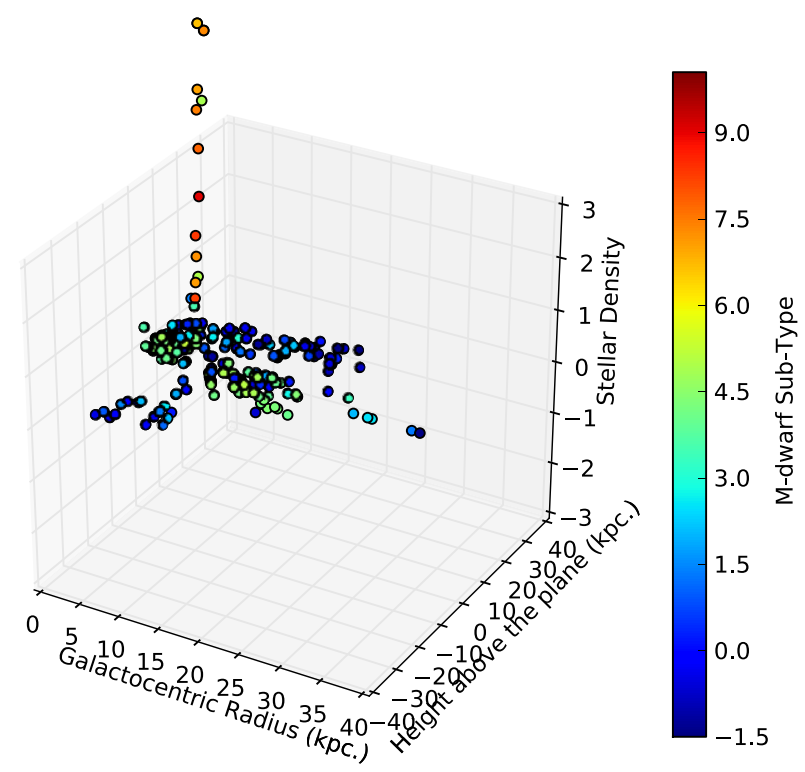

Figure 23. Computed volume for each M-dwarf as a function of Galactocentric radius, height above the plane. M-dwarf types as inferred from their optical-NIR color.

(A color version of this figure is available in the online journal.)

more uncertain. This field contains $67 \mathrm{M}$-dwarf candidates, but these are not used for the following analysis.

Figure 23 shows the distribution of radius and height above the disk for the 274 stars in the BoRG survey with their corresponding number density and type. The fainter late-subtype M-dwarfs are in a localized thin disk, and the bright earlysubtype (e.g., M0) correspond more closely to the thick disk component.

We assume that the Galactic disk has the following parametric shape:

$$
\rho(R, z)=\rho_{0} \exp (-R / h) \operatorname{sech}^{2}\left(\frac{z}{z_{0}}\right),
$$

where $\rho(R, z)$ is the dwarf number density in a point in the disk, $\rho_{0}$ is the central number density, $R$ is Galactocentric radius, $h$ is the scale-length, $z$ is height above the plane, and $z_{0}$ is the scale-height of the disk.

To collapse Figure 23, we weight the volume densities with the exponential part of this equation because we aim to infer the vertical structure first, i.e., we assume a single scale-length and calculate $\exp (-R / h)$ as a weight for each dwarf volume density. In Figure 24, the volume densities have all been scaled this way, assuming the scale-length $(h)$ of the thin disk from Juric et al. (2008) of $2.6 \mathrm{kpc}$. They found a scale-length for the thick disk of $h=2.9 \mathrm{kpc}$. Alternatively, Bensby et al. (2011) found a thin-disk scale-length of $h=3.8 \mathrm{kpc}$ and $h=2.0$ for the thick disk, corroborated by Cheng et al. (2012) and the trend of stellar population and scale-length in Bovy et al. (2012). We use the Bensby et al. thin disk scale-length for dwarfs found below $500 \mathrm{pc}$ and the thick disk one for those more than $500 \mathrm{pc}$ out of the plane. The implicit assumption in this renormalization is that the scale-height does not change with radius, which is observed in external galaxies seen edge-on (Comerón et al. 2011a, 2011b, 2011c; D. Streich et al., in preparation).

Figure 24 shows more clearly how the later-type M-dwarfs (M4-8) in the BoRG fields are concentrated in the thin disk and the earlier types (M0-3) probe both thin and thick disks. It also shows that there may be several disk components in early types with different scale-heights above and below the plane of 


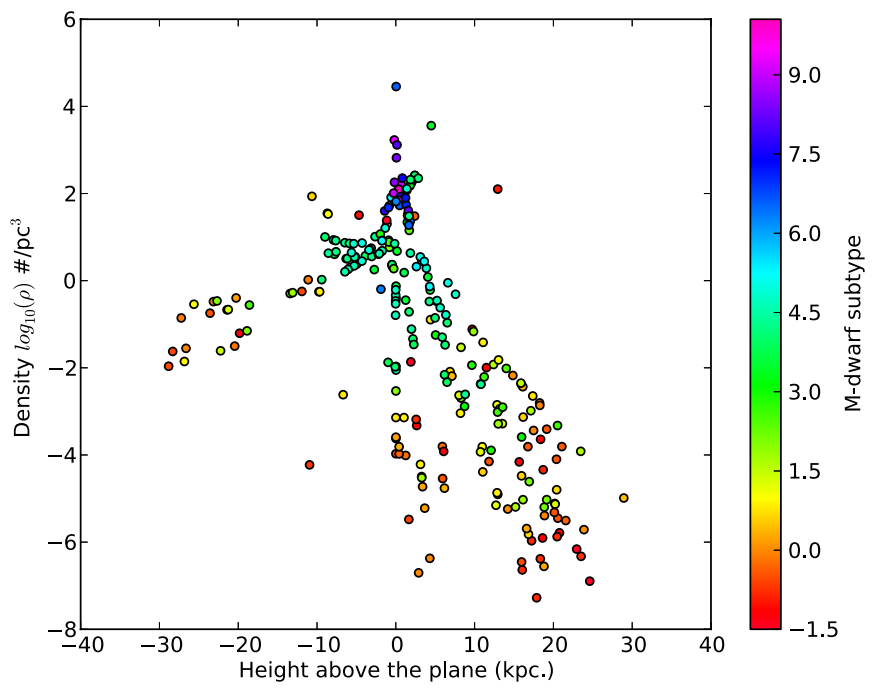

Figure 24. Volume density of M-dwarfs as a function as height above the plane of the Milky Way disk. The volume densities were normalized with a scale-length of $2.6 \mathrm{kpc}$. Points are color-coded by the M-dwarf subtype.

(A color version of this figure is available in the online journal.)

Table 10

The Vertical Profile Fits to the Full Sample of Identified M-dwarfs

\begin{tabular}{lrc}
\hline \hline $\begin{array}{l}\text { M-type } \\
\left({ }^{\circ}\right)\end{array}$ & $\begin{array}{c}\rho_{0} \\
\left(\# / \mathrm{pc}^{3}\right)\end{array}$ & $\begin{array}{c}z_{0} \\
(\mathrm{kpc})\end{array}$ \\
\hline 0 & 4.25 & $22.85 \pm 32.92$ \\
1 & 8.44 & $15.50 \pm 14.33$ \\
2 & 5.29 & $12.62 \pm 9.22$ \\
3 & 373.12 & $10.69 \pm 14.41$ \\
4 & 53.93 & $-5.17 \pm 2.74$ \\
5 & 27.27 & $2.97 \pm 1.39$ \\
6 & 65.78 & $1.24 \pm 0.42$ \\
7 & 14185.51 & $0.28 \pm 0.41$ \\
8 & 1066.36 & $0.28 \pm 0.12$ \\
\hline
\end{tabular}

the disk. This would explain the north-south differential noted earlier by Pirzkal et al. (2009).

First we perform a naive fit on the vertical distribution of M-dwarfs as a function of subtype: we fit the $\operatorname{sech}^{2}\left(z / z_{0}\right)$ to their densities for each subtype, disregarding the different sampling or the existence of more than a single disk component. Fit parameters $\rho_{0}$ and $z_{0}$ are listed for each M-dwarf type in Table 10. The fit to the M4 dwarfs distribution is not a physical solution. Figure 25 shows the best fits to the densities, and Figures 26 and 27 show the central density and scale-height as a function of type.

The central densities in Figure 26 are not scaled by the central volume density of dwarfs. For comparison, we plot the volume densities computed from the local number of M-dwarfs compiled in Reid et al. (2008), their Figure 7, which is based on the numbers reported in Reid et al. $(2004,2007)$ and Cruz et al. (2007) for the latest M-dwarf types (M7-10). Table 11 lists the local densities as a function of M-dwarf type. We only note that both stay relatively constant with M-dwarf subtype.

Figure 27 shows the dependence of scale-height, $z_{0}$, with M-dwarf subtype. The progressive decline in scale-height with subtype is in part due to the different heights sampled. Early types are a mix of thin and thick disk, whereas later types were only found in the thin disk.

Bovy et al. (2012) argue that the different metallicity (and hence age) populations of the Milky Way all have their own

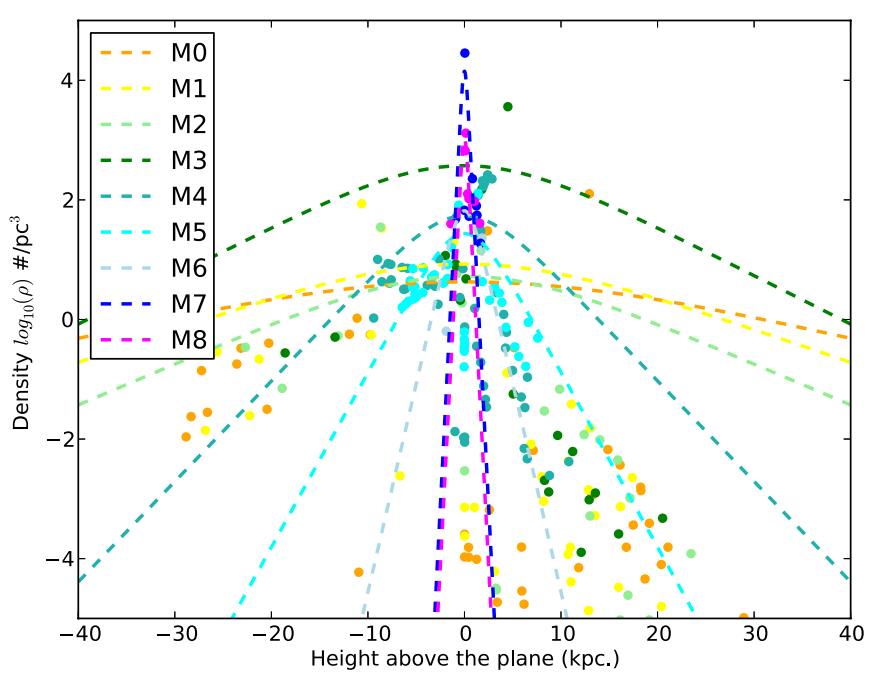

Figure 25. Volume density of M-dwarfs as a function as height above the plane of the Milky Way disk. Points are grayscale-coded by the M-dwarf subtype with best fits shown for each M-dwarf type.

(A color version of this figure is available in the online journal.)

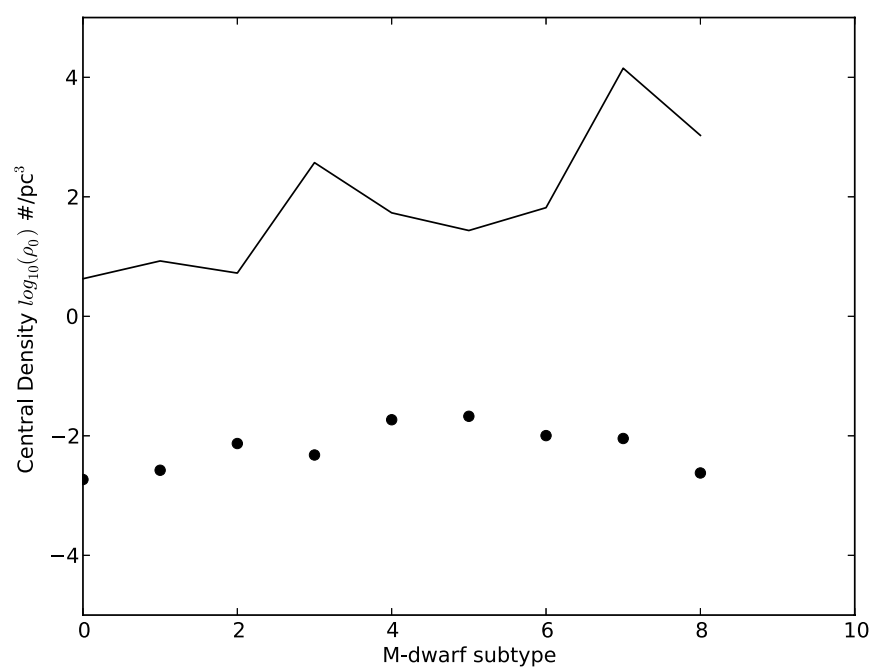

Figure 26. Central density of the best fit as a function of the M-dwarf type. The black circles are the values from Reid et al. (2008).

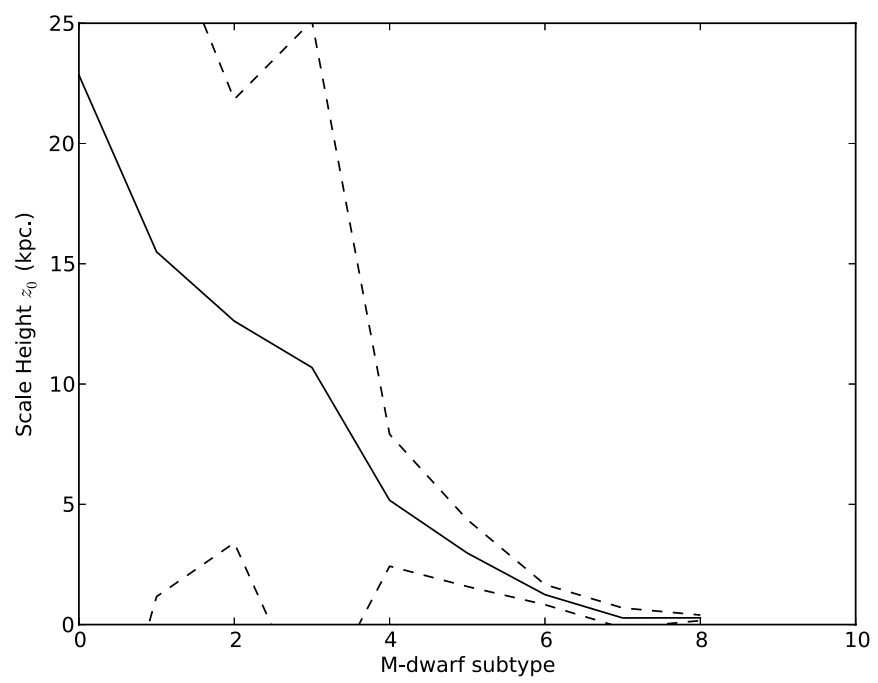

Figure 27. Scale-height of the best fit as a function of the M-dwarf type. Dashed lines are the uncertainty in the fit. 

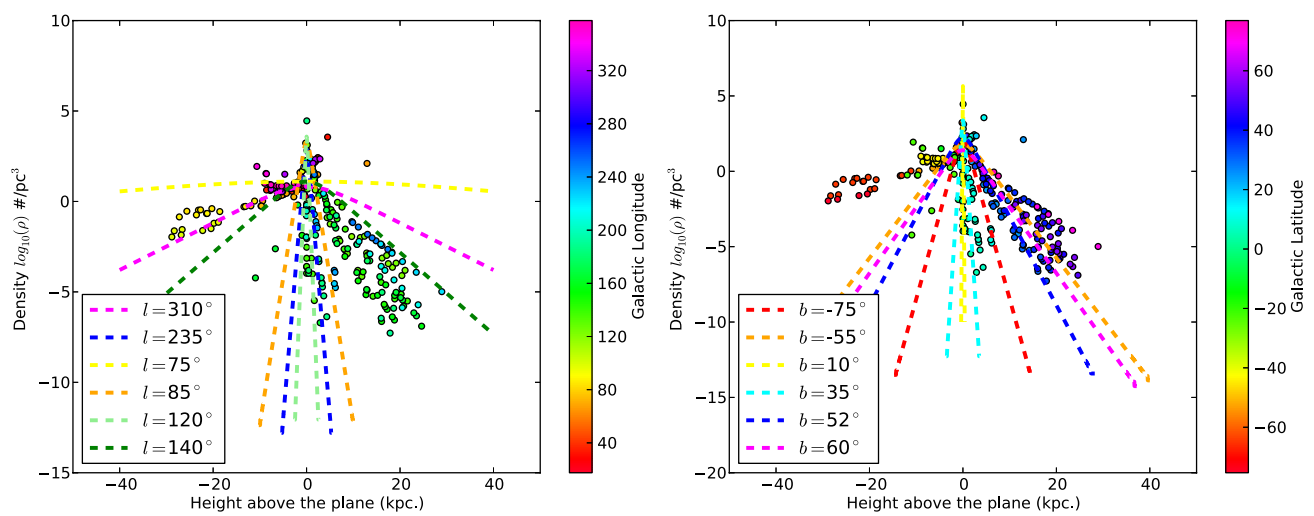

Figure 28. Volume density of M-dwarfs as a function of height above the plane of the Milky Way disk. Points are color-coded by the dwarf's Galactic longitude (left) and latitude (right panel).

(A color version of this figure is available in the online journal.)

Table 11

Local Dwarf Density from Figure 8 in Reid et al. (2008) and Cruz et al. (2007)

\begin{tabular}{lcc}
\hline \hline Subtype & Number within 20 pc & \\
\hline & Reid et al. 2008 & \\
0 & 35 & \\
1 & 50 & \\
2 & 140 & \\
3 & 90 & \\
4 & 350 & \\
5 & 400 & 21 \\
6 & 190 & 21 \\
7 & 170 & 11 \\
8 & 45 & 3 \\
9 & 60 & \\
10 & 42 & \\
\hline
\end{tabular}

distinct and unique scale-length and scale-height. They find that older populations are more concentrated (shorter scalelength) but the most vertically distributed (greatest scale-height). Similar results were found by Bensby et al. (2011) (using a thin-thick disk terminology) and Cheng et al. (2012). The trend between scale-height and M-dwarf subtype in Figure 27 fits with this general picture that there are not distinct thick and thin disks but a gradual transition in scales with stellar population. Because the majority of our fields are high Galactic latitude, we lack the statistics to perform a double fit (vertical and radial). We note, however, that our use of the Bensby et al. (2011) thick- and thin-disk scale-lengths improved the vertical fits substantially over a single scale-length solution.

There are several "spurs" of stars in the height-density plot of Figure 25. To investigate if these correspond to specific areas in the Milky Way, we replot the height-density plot for all M-dwarfs, coded with the Galactic position in Figure 28. Substructures become visible as a function of Galactic longitude. For example, the upturn in M-dwarfs at 0 to $-10 \mathrm{kpc}$ above the plane is due to a few fields near $l \geqslant 310^{\circ}$ and $b=-40^{\circ}$. The longitude position strongly suggests the Milky Way bulge as the origin of the additional M-dwarfs, but the latitude is quite low for much of the effect of the Galactic bulge.

To characterize the different scale-heights implied by the spurs in Figure 28, we selected Galactic longitude or latitude sections for a $\operatorname{sech}^{2}$ fit, summarized in Tables 12 and 13. The $\operatorname{sech}^{2}$ fits to these cuts show an expected variety of $z_{0}$ values. Given the vertical extent that the observations probe, contributions from stellar halo components are likely included
Table 12

The Fits to Selected Galactic Latitude Cuts

\begin{tabular}{lrc}
\hline \hline$b$ & $\rho_{0}$ & \\
$\left({ }^{\circ}\right)$ & $\left(\# / \mathrm{pc}^{3}\right)$ & $\begin{array}{c}z_{0} \\
(\mathrm{kpc})\end{array}$ \\
\hline-75.00 & 140.03 & $0.77 \pm 0.00$ \\
-55.00 & 60.60 & $2.14 \pm 0.07$ \\
10.00 & 451455.50 & $0.02 \pm 0.01$ \\
35.00 & 2394.64 & $0.19 \pm 0.00$ \\
52.00 & 172.83 & $1.48 \pm 0.27$ \\
60.00 & 25.13 & $1.98 \pm 0.01$ \\
\hline
\end{tabular}

Table 13

The Fits to Selected Galactic Longitude Cuts

\begin{tabular}{lrc}
\hline \hline$l$ & $\rho_{0}$ & \\
$\left({ }^{\circ}\right)$ & $\left(\# / \mathrm{pc}^{3}\right)$ & $\begin{array}{c}z_{0} \\
(\mathrm{kpc})\end{array}$ \\
\hline 310.00 & 6.45 & $6.68 \pm 0.38$ \\
235.00 & 716.35 & $0.28 \pm 0.00$ \\
75.00 & 12.64 & $32.24 \pm 77.73$ \\
85.00 & 1921.33 & $0.54 \pm 0.00$ \\
120.00 & 3875.14 & $0.13 \pm 0.01$ \\
140.00 & 12.58 & $3.84 \pm 3.12$ \\
\hline
\end{tabular}

(similar to the Sagittarius stream in Section 6.2). For example, the spur of stars at $20 \mathrm{kpc}$ at $l=-75^{\circ}$ and $b=-75^{\circ}$ (yellow and red points in Figure 28, left and right panels, respectively) is at a higher density than one would expect from the different latitude observations at $l=120^{\circ}$ (green point in Figure 28, left panel). However, that spur is the result of a single field, and this may be simple cosmic variance in our counts. To reliably detect halo substructure or even typical scales using M-dwarfs, one needs to image a much greater area of sky continuously (i.e., with EUCLID).

\subsection{L-Dwarfs and T-Dwarfs}

We do find a number of stellar objects in the $\mathrm{L}$ and $\mathrm{T}$ color selection criteria from Ryan et al. (2011). There is one object brighter than 24 mag in $F 125 \mathrm{~W}$ in these fields that fall into the T-dwarf box. This is not the sole T-dwarf identified by Ryan et al. (2011), which is a 25.08 mag object. The color-color selection also identifies 30 L-dwarfs in the BoRG survey. Table 14 lists their properties. Table 15 lists the properties of the single $m<24$ T-dwarf stars in BoRG. Neither T- nor L-dwarfs have a reasonably well-calibrated relation between optical-nearinfrared color and subtype (see, e.g., Burningham et al. 2013) 
Table 14

The 30 L-dwarfs Identified in BoRG

\begin{tabular}{|c|c|c|c|c|c|c|c|c|}
\hline ID & R.A. & Decl. & $l$ & $b$ & $m_{F 098 M}$ & $m_{F 125 W}$ & $m_{F 125 W}$ & $m_{F 606 W}$ \\
\hline borg_0110-0224.1010.0 & 17.537240 & -2.409470 & 17.537240 & -2.409470 & $24.7 \pm 0.1$ & $23.7 \pm 0.0$ & $23.5 \pm 0.0$ & $25.3 \pm 0.1$ \\
\hline borg_0439-5317.882.0 & 69.836642 & -53.263207 & 69.836642 & -53.263207 & $24.0 \pm 0.0$ & $22.7 \pm 0.0$ & $22.2 \pm 0.0$ & $24.8 \pm 0.9$ \\
\hline borg_0540-6409.2993.0 & 84.876906 & -64.132531 & 84.876906 & -64.132531 & $25.0 \pm 0.1$ & $23.8 \pm 0.0$ & $23.4 \pm 0.0$ & $26.8 \pm 0.6$ \\
\hline borg_0751+2917.567.0 & 117.684321 & 29.285619 & 117.684321 & 29.285619 & $19.5 \pm 0.0$ & $17.8 \pm 0.0$ & $17.6 \pm 0.0$ & $19.6 \pm 0.0$ \\
\hline borg_0808+3946.457.0 & 122.094091 & 39.759095 & 122.094091 & 39.759095 & $25.0 \pm 0.1$ & $23.9 \pm 0.0$ & $23.5 \pm 0.0$ & $26.9 \pm 0.6$ \\
\hline borg_0819+4911.264.0 & 124.831743 & 49.175806 & 124.831743 & 49.175806 & $23.9 \pm 0.0$ & $22.8 \pm 0.0$ & $22.4 \pm 0.0$ & $27.9 \pm 1.6$ \\
\hline borg_0906+0255.462.0 & 136.409407 & 2.923645 & 136.409407 & 2.923645 & $24.4 \pm 0.0$ & $23.3 \pm 0.0$ & $22.8 \pm 0.0$ & $27.6 \pm 0.8$ \\
\hline borg_0909+0002.748.0 & 137.292821 & -0.012257 & 137.292821 & -0.012257 & $24.5 \pm 0.1$ & $23.2 \pm 0.0$ & $22.8 \pm 0.3$ & $26.6 \pm 0.4$ \\
\hline borg_0914+2822.673.0 & 138.558022 & 28.364206 & 138.558022 & 28.364206 & $24.8 \pm 0.1$ & $23.5 \pm 0.0$ & $23.1 \pm 0.0$ & $26.3 \pm 0.3$ \\
\hline borg_0926+4426.581.0 & 141.355847 & 44.425445 & 141.355847 & 44.425445 & $24.3 \pm 0.0$ & $23.1 \pm 0.0$ & $22.6 \pm 0.0$ & $25.2 \pm 0.9$ \\
\hline borg_1103-2330.623.0 & 165.819910 & -23.506010 & 165.819910 & -23.506010 & $23.9 \pm 0.1$ & $22.9 \pm 0.0$ & $22.5 \pm 0.0$ & $25.7 \pm 0.2$ \\
\hline borg_1111+5545.1125.0 & 167.747157 & 55.771759 & 167.747157 & 55.771759 & $23.6 \pm 0.0$ & $22.5 \pm 0.0$ & $22.1 \pm 0.0$ & $26.6 \pm 0.3$ \\
\hline borg_1153+0056.247.0 & 178.200215 & 0.922794 & 178.200215 & 0.922794 & $23.9 \pm 0.0$ & $22.9 \pm 0.0$ & $22.4 \pm 0.0$ & $26.2 \pm 0.3$ \\
\hline borg_1230+0750.2419.0 & 187.471709 & 7.820117 & 187.471709 & 7.820117 & $23.9 \pm 0.1$ & $22.8 \pm 0.0$ & $22.3 \pm 0.0$ & $26.2 \pm 0.3$ \\
\hline borg_1301+0000.1067.0 & 195.326828 & 0.013297 & 195.326828 & 0.013297 & $23.5 \pm 0.1$ & $22.2 \pm 0.0$ & $21.8 \pm 0.0$ & $24.3 \pm 0.9$ \\
\hline borg_1337+0028.1117.0 & 204.205278 & -0.445870 & 204.205278 & -0.445870 & $24.3 \pm 0.2$ & $23.1 \pm 0.0$ & $22.7 \pm 0.0$ & $25.3 \pm 0.9$ \\
\hline borg_1524+0954.24.0 & 231.044740 & 9.885957 & 231.044740 & 9.885957 & $24.9 \pm 0.1$ & $23.0 \pm 0.1$ & $22.8 \pm 0.1$ & $25.3 \pm 1.0$ \\
\hline borg_1632+3733.65.0 & 248.069017 & 37.540353 & 248.069017 & 37.540353 & $24.5 \pm 0.0$ & $23.3 \pm 0.0$ & $22.9 \pm 0.0$ & $28.5 \pm 2.1$ \\
\hline borg_1632+3733.349.0 & 248.080963 & 37.550065 & 248.080963 & 37.550065 & $23.5 \pm 0.0$ & $22.4 \pm 0.0$ & $22.0 \pm 0.1$ & $27.1 \pm 1.4$ \\
\hline borg_1632+3733.1325.0 & 248.076265 & 37.572819 & 248.076265 & 37.572819 & $24.3 \pm 0.0$ & $23.0 \pm 0.1$ & $22.5 \pm 0.0$ & $26.7 \pm 0.5$ \\
\hline borg_2132+1004.284.0 & 323.058751 & 10.068991 & 323.058751 & 10.068991 & $16.8 \pm 0.0$ & $15.0 \pm 0.0$ & $14.7 \pm 0.0$ & $17.2 \pm 0.9$ \\
\hline borg_2132+1004.332.0 & 323.060235 & 10.059801 & 323.060235 & 10.059801 & $21.2 \pm 0.0$ & $19.9 \pm 0.0$ & $19.7 \pm 0.0$ & $22.2 \pm 0.9$ \\
\hline borg_2155-4411.227.0 & 328.831076 & -44.185153 & 328.831076 & -44.185153 & $23.5 \pm 0.0$ & $22.5 \pm 0.0$ & $22.2 \pm 0.0$ & $24.8 \pm 0.9$ \\
\hline borg_2155-4411.974.0 & 328.809893 & -44.164189 & 328.809893 & -44.164189 & $24.3 \pm 0.0$ & $23.0 \pm 0.0$ & $22.5 \pm 0.0$ & $25.1 \pm 0.9$ \\
\hline borg_2351-4332.1314.0 & 357.668728 & -43.514053 & 357.668728 & -43.514053 & $25.2 \pm 0.1$ & $23.9 \pm 0.0$ & $23.5 \pm 0.0$ & $26.0 \pm 0.9$ \\
\hline borg_0456-2203.920.0 & 73.945566 & -22.049396 & 73.945566 & -22.049396 & $24.6 \pm 0.1$ & $23.4 \pm 0.0$ & $23.2 \pm 0.0$ & $24.4 \pm 0.0$ \\
\hline borg_1059+0519.644.0 & 164.717943 & 5.301323 & 164.717943 & 5.301323 & $24.4 \pm 0.0$ & $23.1 \pm 0.0$ & $22.6 \pm 0.0$ & $26.5 \pm 0.3$ \\
\hline borg_1118-1858.1915.0 & 169.392755 & -18.972497 & 169.392755 & -18.972497 & $24.8 \pm 0.0$ & $23.8 \pm 0.0$ & $23.3 \pm 0.0$ & $26.1 \pm 0.1$ \\
\hline borg_1459+7146.1298.0 & 224.751450 & 71.780685 & 224.751450 & 71.780685 & $24.8 \pm 0.2$ & $23.5 \pm 0.0$ & $23.1 \pm 0.0$ & $25.7 \pm 0.1$ \\
\hline borg_2132-1202.6461.0 & 322.941469 & -12.021619 & 322.941469 & -12.021619 & $24.6 \pm 0.1$ & $23.4 \pm 0.0$ & $23.0 \pm 0.0$ & $26.5 \pm 0.4$ \\
\hline
\end{tabular}

Table 15

The 1 T-dwarfs Identified in BoRG

\begin{tabular}{|c|c|c|c|c|c|c|c|c|}
\hline ID & R.A. & Decl. & $l$ & $b$ & $m_{F 098 M}$ & $m_{F 125 W}$ & $m_{F 125 W}$ & $m_{F 606 W}$ \\
\hline borg_0540-6409.1929.0 & 84.862502 & -64.152446 & 84.862502 & -64.152446 & $22.9 \pm 0.0$ & $21.9 \pm 0.0$ & $22.2 \pm 0.0$ & $21.5 \pm 0.0$ \\
\hline
\end{tabular}

Table 16

The 274 M-dwarfs Identified in BoRG

\begin{tabular}{|c|c|c|c|c|c|c|c|c|c|c|c|c|c|}
\hline ID & R.A. & Decl. & $l$ & $b$ & $m_{F 098 M}$ & $m_{F 125 W}$ & $m_{F 160 W}$ & $m_{F 606 W}$ & Mtype & Modulus & Dist & Height & Radius \\
\hline borg_0110-0224.551.0 & 17.501759 & -2.418265 & 17.501759 & -2.418265 & $23.8 \pm 0.0$ & $23.5 \pm 0.0$ & $23.3 \pm 0.0$ & $25.2 \pm 0.1$ & $-0.0 \pm 0.2$ & 16.48 & 19.74 & -0.81 & 27.33 \\
\hline borg_0110-0224.719.0 & 17.503586 & -2.415663 & 17.503586 & -2.415663 & $24.3 \pm 0.1$ & $24.0 \pm 0.0$ & $23.9 \pm 0.0$ & $25.8 \pm 0.1$ & $5.1 \pm 1.0$ & 16.73 & 22.16 & -0.91 & 29.63 \\
\hline borg_0110-0224.820.0 & 17.528020 & -2.411389 & 17.528020 & -2.411389 & $19.7 \pm 0.0$ & $19.5 \pm 0.0$ & $19.4 \pm 0.0$ & $22.8 \pm 0.0$ & $0.6 \pm 0.2$ & 8.59 & 0.52 & 0.01 & 9.00 \\
\hline borg_0110-0224.1016.0 & 17.553100 & -2.408888 & 17.553100 & -2.408888 & $24.5 \pm 0.1$ & $23.9 \pm 0.0$ & $23.7 \pm 0.0$ & $25.4 \pm 0.1$ & $6.0 \pm 0.7$ & 17.18 & 27.26 & -1.12 & 34.49 \\
\hline borg_0110-0224.1414.0 & 17.559120 & -2.400783 & 17.559120 & -2.400783 & $24.1 \pm 0.1$ & $23.7 \pm 0.1$ & $23.4 \pm 0.0$ & $24.4 \pm 0.1$ & $11.6 \pm 0.2$ & 17.26 & 28.31 & -1.16 & 35.49 \\
\hline borg_0110-0224.1529.0 & 17.564067 & -2.398369 & 17.564067 & -2.398369 & $24.2 \pm 0.1$ & $23.9 \pm 0.0$ & $23.8 \pm 0.1$ & $25.8 \pm 0.2$ & $2.0 \pm 0.3$ & 16.67 & 21.59 & -0.88 & 29.08 \\
\hline borg_0110-0224.2048.0 & 17.516425 & -2.384891 & 17.516425 & -2.384891 & $22.8 \pm 0.0$ & $22.5 \pm 0.0$ & $22.2 \pm 0.0$ & $24.4 \pm 0.0$ & $5.8 \pm 0.4$ & 15.23 & 11.11 & -0.44 & 19.09 \\
\hline borg_0110-0224.2599.0 & 17.523929 & -2.371366 & 17.523929 & -2.371365 & $22.3 \pm 0.0$ & $22.2 \pm 0.0$ & $21.9 \pm 0.0$ & $23.5 \pm 0.0$ & $2.6 \pm 0.5$ & 15.46 & 12.33 & -0.48 & 20.26 \\
\hline borg_0110-0224.2683.0 & 17.528492 & -2.352761 & 17.528492 & -2.352761 & $24.4 \pm 0.1$ & $23.8 \pm 0.0$ & $23.5 \pm 0.0$ & $26.1 \pm 0.2$ & $-0.5 \pm 0.3$ & 15.47 & 12.40 & -0.48 & 20.32 \\
\hline borg_0110-0224.3274.0 & 17.527112 & -2.365442 & 17.527112 & -2.365442 & $21.5 \pm 0.0$ & $21.2 \pm 0.0$ & $20.9 \pm 0.0$ & $23.0 \pm 0.0$ & $7.3 \pm 0.0$ & 14.22 & 6.99 & -0.26 & 15.16 \\
\hline
\end{tabular}

(This table is available in its entirety in a machine-readable form in the online journal. A portion is shown here for guidance regarding its form and content.)

to allow for a distance estimate similar to the M-dwarfs, nor do we have the statistics in hand to infer a scale-height for these objects.

Wilkins et al. (2014) reiterate that L- and T-dwarfs pose a risk of contaminating high-redshift $(z \sim 7)$ color-selected samples and show how some dwarf spectra may even masquerade as objects with emission lines. They generated their color selections similar to Ryan et al. (2011), and the boxes in Figure 12 do generally agree with the derived colors. Wilkins et al. (2014) claim HST morphological information is insufficient to cull L- and
T-dwarfs from a high-redshift galaxy sample. We have shown that dwarfs can be unequivocally identified by their stellar morphology using simple tools such as SEXTRACTOR down to $24 \mathrm{AB}$ mag in undithered WFC3 data, and a reliable stargalaxy separation is not only crucial for high-redshift studies but very likely possible down to much lower luminosities. The second issue is the surface density of these later dwarf types. For the first time, we can give an indication for random high-latitude, spacebased observations based on the number of L-dwarfs found (Table 14). Figure 29 shows the surface density of L-dwarfs 


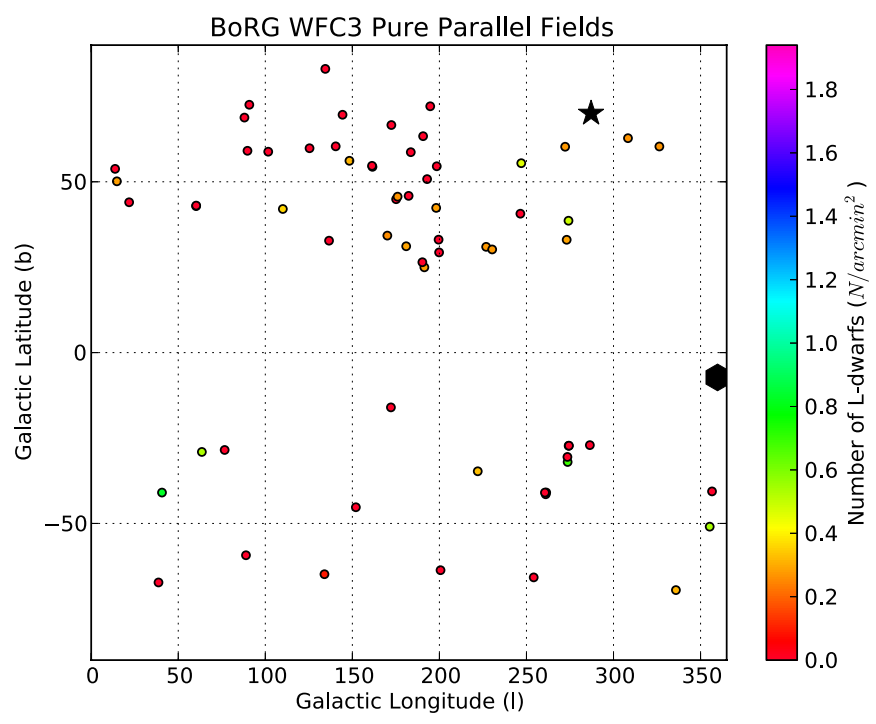

Figure 29. Distribution of BoRG fields with the number of L-dwarfs indicated. Other symbols identical to Figure 21.

(A color version of this figure is available in the online journal.)

selected by our morphology and color criteria. It shows a WFC3 exposure has $<1$ L-dwarf that can be selected against morphologically. Extrapolating to lower luminosities (including improved stargalaxy separation), the contamination problem may not be as dire as predicted. For example, Wilkins et al. (2014) assume that the local brown-dwarf density (0.015 stars $\left.\mathrm{pc}^{-3}\right)$ holds throughout all of the Milky Way components (thin and thick disks and halo). However, the spatial density drops exponentially with height above the place. If L- and T-dwarfs follow a similar scale-height as the M-dwarfs examined here $\left(z_{0} \sim 300 \mathrm{pc}\right)$, then contaminating dwarf stars at $1-2 \mathrm{kpc}$ distance will be very rare, even before morphological selection.

\section{CONCLUSIONS}

1. Stars can be selected best using SEXTRACTOR's half-light radius $\left(r_{50}\right)$, corroborating previous identifications (Figures 8 and 17$)$.

2. M-dwarfs can be reliably subtyped using near-infrared and optical color (Figure 14, Table 16).

3. Relatively more early-type M-dwarfs are identified in northern fields as opposed to southern Galactic ones. This confirms the dichotomy Pirzkal et al. (2009) noted (Table 9).

4. We report an overdensity in one of our fields, borg_1230+0750, consistent with the M-dwarf members of the Sagittarius stream at a distance of 20-25 kpc (Figure 22).

5. A naive, single-component fit of the vertical distribution of M-dwarfs shows a steady decline of scale-height, $z_{0}$, with M-dwarf subtype (Figure 27).

6. The grouping of spurs of stars at different Galactic coordinates strongly hints at substructure in the Milky Way disk/halo of M-dwarfs (Figure 28).

\section{FUTURE WORK}

Future work with WFC3 (pure-parallel) programs on Milky Way dwarfs will use the ever-increasing number of sight-lines available to build a comprehensive catalog of M-, L-, and T-dwarfs belonging to the thin and thick disks and stellar halo of our Milky Way. New pure-parallel observational programs with $H S T /$ WFC3 are needed to expand the number of sightlines and improve statistics. At lower Galactic latitudes, this could easily be achieved with lower integration times than the BoRG or HIPPIES programs. Once such a proposed program is completed fully, the total tally of M-dwarfs should give a first idea of the size and shape of the Milky Way disks, thin and thick, and depending on the total statistics, the Galactic halo.

In parallel, spectroscopic identification of Milky Way dwarf stars can be done with the HST-3D near-infrared grism spectra (e.g., Brammer et al. 2012). The ongoing imaging and grism campaigns on the CANDELS fields (Koekemoer et al. 2011; Grogin et al. 2011) will improve the calibration of the photometric identifications of Galactic dwarfs in the pure-parallel surveys as well as add additional statistics to the star counts.

The general technique of morphologically identifying stars in space-based imaging, identifying dwarfs from near-infrared colors and subsequently subtyping these using an optical-nearinfrared color will be perfectly suited for the EUCLID mission (Laureijs et al. 2011). The wide-band optical filter can be used to morphologically identify the stars and the three nearinfrared filters to home in on the Galactic dwarfs. Finally, the optical-NIR color and complementary grism spectroscopy can then be used to subtype these dwarfs. With the wide survey area to faint point source sensitivity (all-sky $24 \mathrm{mag}, 26 \mathrm{mag}$ in deep fields) the EUCLID mission will yield an accurate measure of the shape and size of our Milky Way in subsolar dwarfs as well as an accurate census of halo substructure similar to the Sagittarius stream. Limiting factors may be the PSF in the near-infrared, leading to confusion issues, and the width of the optical filter, complicating subtyping. However, the two independent-angle grism spectra of all the objects in the field should unequivocally identify the Galactic dwarf stars.

Milky Way dwarfs are unlikely to be the focus of James Webb Space Telescope (JWST) observations, but they will certainly feature in them. The ubiquity of M-dwarfs in the BoRG fields points to their possible use as fine guidance for JWST image and NIRspec imaging/MOS registration.

The authors would like to thank N. Reid and K. Cruz for their help with the local density of M-dwarfs and R. Benjamin for useful discussions on Galactic substructures. The lead author thanks the European Space Agency for the Fellowship program and its support. We acknowledge the support of HST Archival grant number AR-12134, General Observer Grants GO-11700, GO-12572, and GO-12905, and the European Space Agency for support of this work. M.T. was partially supported by the European Commission through the Marie Curie Career Integration Fellowship PCIG12-GA-2012-333749. This work is based in part on observations taken by the CANDELS Multi-Cycle Treasury Program with the NASA/ESA $H S T$, which is operated by the Association of Universities for Research in Astronomy, Inc., under NASA contract NAS5-26555. This research has made use of the NASA/IPAC Extragalactic Database (NED), which is operated by the Jet Propulsion Laboratory, California Institute of Technology, under contract with the National Aeronautics and Space Administration. This research has made use of NASA's Astrophysics Data System.

\section{REFERENCES}

Bahcall, J. N. 1986, ARA\&A, 24, 577

Beckwith, S. V. W., Stiavelli, M., Koekemoer, A. M., et al. 2006, AJ, 132, 1729 Belokurov, V., Koposov, S. E., Evans, N. W., et al. 2014, MNRAS, 437, 116 Belokurov, V., Zucker, D. B., Evans, N. W., et al. 2006, ApJL, 642, L137 
Bensby, T., Alves-Brito, A., Oey, M. S., Yong, D., \& Meléndez, J. 2011, ApJL, 735, L46

Bertin, E., \& Arnouts, S. 1996, A\&AS, 117, 393

Bochanski, J. J., Willman, B., West, A. A., Strader, J., \& Chomiuk, L. 2014, ApJ, 147, 76

Bovy, J., Rix, H.-W., Liu, C., et al. 2012, ApJ, 753, 148

Bradley, L. D., Trenti, M., Oesch, P. A., et al. 2012, ApJ, 760, 108

Brammer, G. B., van Dokkum, P. G., Franx, M., et al. 2012, ApJS, 200, 13

Burningham, B., Cardoso, C. V., Smith, L., et al. 2013, MNRAS, 433, 457

Caballero, J. A., Burgasser, A. J., \& Klement, R. 2008, A\&A, 488, 181

Casertano, S., de Mello, D., Dickinson, M., et al. 2000, AJ, 120, 2747

Cheng, J. Y., Rockosi, C. M., Morrison, H. L., et al. 2012, ApJ, 752, 51

Comerón, S., Elmegreen, B. G., Knapen, J. H., et al. 2011a, ApJL, 738, L17

Comerón, S., Elmegreen, B. G., Knapen, J. H., et al. 2011b, ApJ, 741, 28

Comerón, S., Knapen, J. H., Sheth, K., et al. 2011c, ApJ, 729, 18

Cruz, K. L., Reid, I. N., Kirkpatrick, J. D., et al. 2007, AJ, 133, 439

Dressel, L., Wong, M. H., \& Pavlovsky, C. 2010, Wide Field Camera 3 Instrument Handbook, Version 3.0 (Baltimore: STScI)

Fruchter, A. S., \& Hook, R. N. 2002, PASP, 114, 144

Giavalisco, M., Ferguson, H. C., Koekemoer, A. M., et al. 2004, ApJL, 600, L93

Gilmore, G. 1984, MNRAS, 207, 223

Gilmore, G., \& Reid, N. 1983, MNRAS, 202, 1025

Gilmore, G., Wyse, R. F. G., \& Kuijken, K. 1989, ARA\&A, 27, 555

Grogin, N. A., Kocevski, D. D., Faber, S. M., et al. 2011, ApJS, 197, 35

Hawley, S. L., Covey, K. R., Knapp, G. R., et al. 2002, AJ, 123, 3409

Holwerda, B. W. 2005, arXiv:astro-ph/0512139

Holwerda, B. W., González, R. A., Allen, R. J., \& van der Kruit, P. C. 2005, AJ, 129,1396

Jurić, M., Ivezić, Ž., Brooks, A., et al. 2008, ApJ, 673, 864

Kapteyn, J. C. 1922, ApJ, 55, 302

Kilic, M., Mendez, R. A., von Hippel, T., \& Winget, D. E. 2005, ApJ, 633, 1126

King, I., Gilmore, G., \& van der Kruit, P. C., (ed.) 1990, The Milky Way As Galaxy (Mill Valley, CA: University Science Books)
Koekemoer, A. M., Faber, S. M., Ferguson, H. C., et al. 2011, ApJS, 197, 36

Laureijs, R., Amiaux, J., Arduini, S., et al. 2011, arXiv:1110.3193

Leauthaud, A., Massey, R., Kneib, J., et al. 2007, ApJS, 172, 219

Majewski, S. R. 1993, ARA\&A, 31, 575

Majewski, S. R., Skrutskie, M. F., Weinberg, M. D., \& Ostheimer, J. C. 2003, ApJ, 599, 1082

Newberg, H. J., Yanny, B., Rockosi, C., et al. 2002, ApJ, 569, 245

Pirzkal, N., Burgasser, A. J., Malhotra, S., et al. 2009, ApJ, 695, 1591

Pirzkal, N., Sahu, K. C., Burgasser, A., et al. 2005, ApJ, 622, 319

Pirzkal, N., Xu, C., Malhotra, S., et al. 2004, ApJS, 154, 501

Reid, I. N., Cruz, K. L., \& Allen, P. R. 2007, AJ, 133, 2825

Reid, I. N., Cruz, K. L., Allen, P. R., et al. 2004, AJ, 128, 463

Reid, I. N., Cruz, K. L., Kirkpatrick, J. D., et al. 2008, AJ, 136, 1290

Ryan, R. E., Hathi, N. P., Jr, Cohen, S. H., \& Windhorst, R. A. 2005, ApJL, 631, L159

Ryan, R. E., Thorman, P. A., Yan, H., et al. 2011, ApJ, 739, 83

Schmidt, K. B., et al. 2014, arXiv:1402.4129

Siegel, M. H., Majewski, S. R., Reid, I. N., \& Thompson, I. B. 2002, ApJ, 578,151

Stanway, E. R., Bremer, M. N., Lehnert, M. D., \& Eldridge, J. J. 2008, MNRAS, 384,348

Steidel, C. C., Giavalisco, M., Dickinson, M., \& Adelberger, K. L. 1996, AJ, 112,352

Trenti, M., Bradley, L. D., Stiavelli, M., et al. 2011, ApJL, 727, L39

Trenti, M., Bradley, L. D., Stiavelli, M., et al. 2012, ApJ, 746, 55

Trenti, M., \& Stiavelli, M. 2008, ApJ, 676, 767

van Dokkum, P. G. 2001, PASP, 113, 1420

Widrow, L. M., Gardner, S., Yanny, B., Dodelson, S., \& Chen, H.-Y. 2012, ApJL, 750, L41

Wilkins, S. M., Stanway, E. R., \& Bremer, M. N. 2014, MNRAS, 439, 1038

Windhorst, R. A., Cohen, S. H., Hathi, N. P., et al. 2011, ApJS, 193, 27

Yan, H., Yan, L., Zamojski, M. A., et al. 2010, arXiv:1010.2261

Yanny, B., \& Gardner, S. 2013, ApJ, 777, 91

Zheng, Z., Flynn, C., Gould, A., Bahcall, J. N., \& Salim, S. 2001, ApJ, 555, 393 


\title{
ERRATUM: "MILKY WAY RED DWARFS IN THE BORG SURVEY; GALACTIC SCALE-HEIGHT AND THE
} DISTRIBUTION OF DWARFS STARS IN WFC3 IMAGING” (2014, ApJ, 788, 77)

\author{
B. W. Holwerda ${ }^{1,2,7,8}$, M. Trenti ${ }^{3}$, W. Clarkson ${ }^{4}$, K. Sahu ${ }^{5}$, L. Bradley ${ }^{5}$, M. Stiavelli ${ }^{5}$, N. Pirzkal ${ }^{5}$, G. De Marchi ${ }^{2}$,

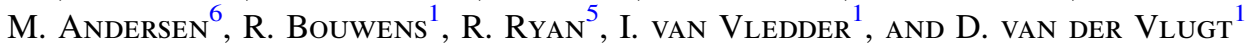 \\ ${ }^{1}$ Leiden Observatory, Leiden University, P.O. Box 9513, 2300 RA Leiden, The Netherlands; holwerda@strw.leidenuniv.nl \\ ${ }^{2}$ European Space Agency, ESA-ESTEC, Keplerlaan 1, 2200 AG Noordwijk, The Netherlands \\ ${ }^{3}$ School of Physics, University of Melbourne, VIC 3010, Australia \\ ${ }^{4}$ Department of Natural Sciences College of Arts, Sciences and Letters, University of Michigan-Dearborn 4901 Evergreen Road, Dearborn, MI:48128, USA \\ ${ }^{5}$ Space Telescope Science Institute, Baltimore, MD 21218, USA \\ ${ }^{6}$ UJF-Grenoble 1/CNRS-INSU, Institut de Planétologie et d'Astrophysique de Grenoble (IPAG) UMR 5274, Grenoble, F-38041, France \\ Received 2015 October 20; revised 2016 February 3; accepted 2016 February 3; published 2016 July 5
}

\section{ABSTRACT}

In the catalog of M-dwarfs presented in Holwerda et al. (2014, H14 hereafter), there is an issue with the conversion from celestial coordinates to Galactic ones, done with PYEPHEM a wrapper around a trusted and vetted library ephermis. Here we present the corrected coordinates (using AsTroPy) and distances based on $\mathrm{AB}$ magnitudes. We have amended the tables and figures accordingly. The relation between vertical scale-height $\left(z_{0}\right)$ and M- dwarf subtype found in H14 is no longer present. We find a scale-height of $600 \mathrm{pc}$ for all types, in part due to the presence of a second Galactic structural component.

\section{MAPPING THE M-DWARF DISTRIBUTION}

We re-computed the Galactic coordinates for the BoRG fields using the AstroPy package (Astropy Collaboration et al. 2013) and the number of M-dwarfs in each, shown in the new Figure 22. The new values for all identified M-dwarfs are listed in Table 14. To estimate the distances to each M-dwarf, we compute the distance modulus from the inferred sub-type (and hence absolute magnitude) and the apparent magnitude in $J_{F 125 W}$ from Hawley et al. (2002), converted from Vega magnitudes to AB. We compute the Galactic radius and height above the plane for all M-dwarfs, based on their Galactic longitude, latitude and the inferred photometric distances, assuming the position of the Sun $27 \mathrm{pc}$. above the plane and $8.5 \mathrm{kpc}$ from the Galactic center.

The net difference with Holwerda et al. (2014, H14 hereafter) is that the vertical distribution observed for these M-dwarfs is much closer to the exponential drop-off one would expect for fields out of the plane of the Galaxy.

Figure 24 shows the vertical distribution of M-dwarfs color-coded by their Galactocentric radius. There is a clear disk component with all the M-dwarfs are $\sim 6-10 \mathrm{kpc}$ from the center of the Milky Way and another component which shows a gradient with radius and is well above the plane of the disk. We now know this component to be the Halo part of the vertical distribution (van Vledder et al. 2016) but it is sometimes treated as the thick disk by other authors (e.g., Ryan \& Reid 2016). This second component is much clearer in the corrected Galactic positions compared to H14. The majority of stars close to the plane of the disk are $\sim 8 \mathrm{kpc}$ from the center of the Galaxy. This is because the majority of BoRG fields are pointing out of the disk at an angle away from the Galactic Center (to avoid confusion with Galactic Objects) and thus probe the vertical extent of the disk close to the position of the Sun.

We assume there is no need to correct the densities in this part of the disk for the exponential decline with radius. Figure 24 does show a radial dependence of the outer parts of the vertical distribution on the radius. Our assumption is that the scale-height does not change significantly with radius, which is observed in external galaxies seen edge-on (Comerón et al. 2011a, 2011a, 2011c, Streich et al. 2016 in preparation).

We assume that the Galactic disk has the following parametric shape:

$$
\rho(R, z)=\rho_{0} \exp (-R / h) \operatorname{sech}^{2}\left(\frac{z}{2 \times z_{0}}\right)
$$

where $\rho(R, z)$ is the dwarf number density in a point in the disk, $\rho_{0}$ is the central number density, $R$ is Galactocentric radius, $h$ is the scale-length, $z$ is height above the plane, and $z_{0}$ is the exponential scale-height of the disk. Figure 25 shows how the later-type M-dwarfs (M4-8) in the BoRG fields are concentrated in the thin disk and the earlier types (M0-3) probe both thin disk and the other component. We fit the $\operatorname{sech}^{2}$ distribution to each photometric subtype, as well as early-, late- and all-types of M-dwarfs, summarized in the new Table 10 and the new Figures 26 and 27.

Figures 26 and 27 are significantly different from the versions in H14 in two ways: the relation between vertical scale-height $\left(z_{0}\right)$ and M- dwarf subtype found in H14 is no longer present in Figure 27. Second, the densities inferred are much closer to the local ( $<20 \mathrm{pc}$ of the Sun) Reid et al. (2004) and Cruz et al. (2007) values (Figure 26). We note there is an offset of $\sim 0.1$ dex between our central density and the one implied by Reid et al. The likely culprit is the additional numbers of M-dwarfs in the secondary

\footnotetext{
7 twitter:benneholwerda.

${ }^{8}$ Research Fellow.
} 


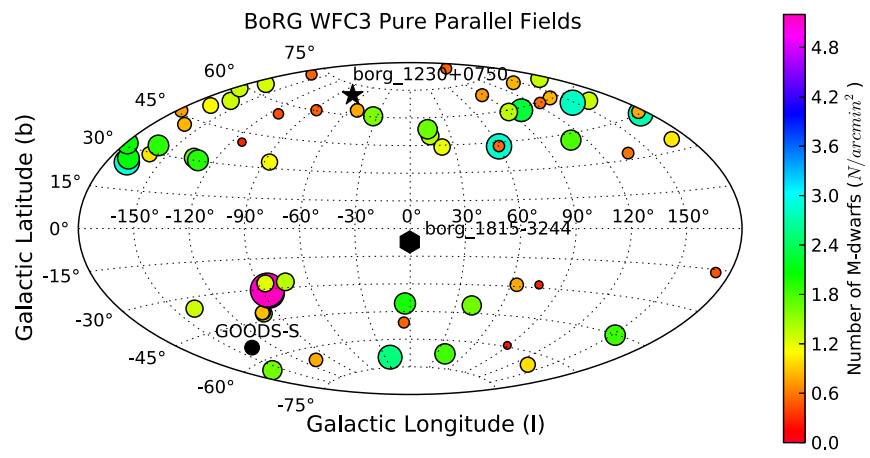

Figure 22. The Aitoff projection of the distribution of BoRG fields with the surface density of M-dwarfs of all types indicated. One field, borg_1230+0750 (star) stands out with 22 M-dwarfs $\left(\sim 5\right.$ stars $\left.\operatorname{arcmin}^{-2}\right)$. We discard borg_1815-3244 (black circle) for its low latitudes and line-of-sight through the plane of the disk and close of the center of the bulge.

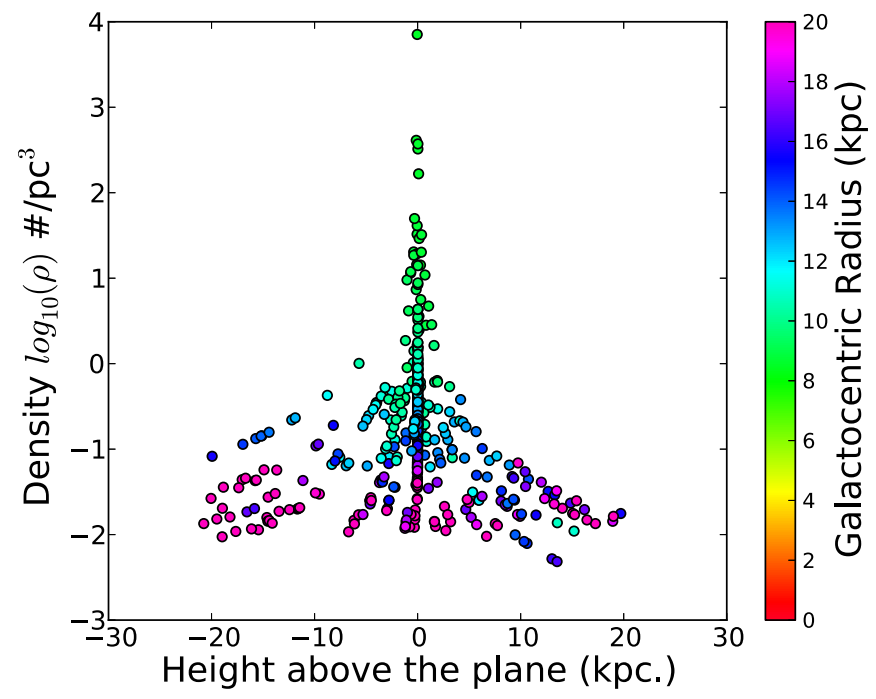

Figure 24. The volume density of M-dwarfs as a function as height above the plane of the Milky Way disk. The volume densities were normalized with a scale-length of $2.6 \mathrm{kpc}$ and a depth of $1 \mathrm{pc}$ at each star's position. Points are color-coded by the inferred Galactic radius. The majority of M-dwarfs are found close to the plane of the disk.

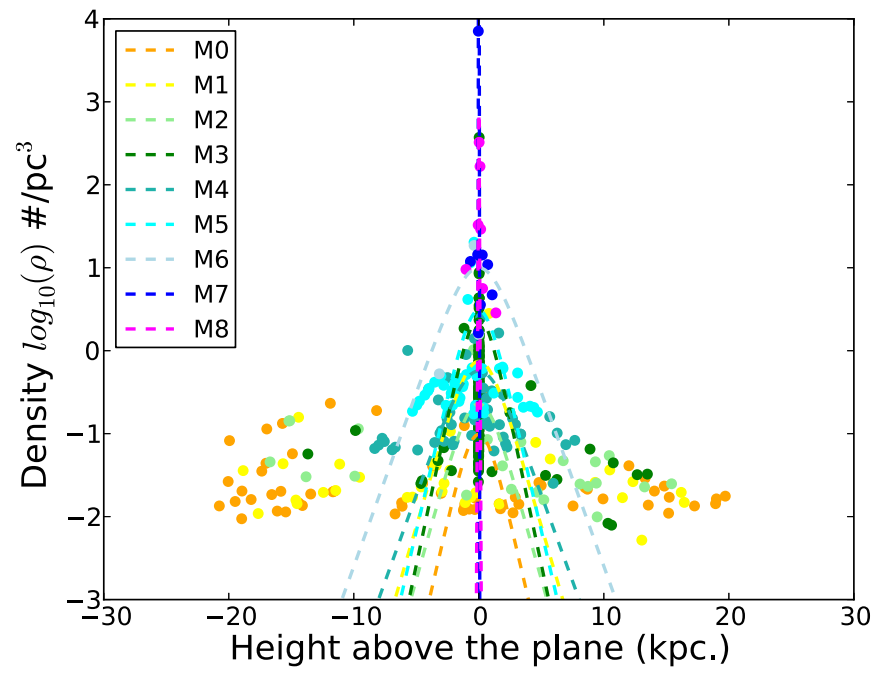

Figure 25. The volume density of M-dwarfs as a function as height above the plane of the Milky Way disk. Points and fits are color-coded by the M-dwarf sub-type.
Table 10

The Vertical Profile Fits to The Full Sample of Identified M-dwarfs

\begin{tabular}{lcc}
\hline \hline $\begin{array}{l}\text { M-type } \\
\text { (o) }\end{array}$ & $\begin{array}{c}\rho_{0} \\
\left(\# / p c^{3}\right)\end{array}$ & $\begin{array}{c}z_{0} \\
(\mathrm{kpc})\end{array}$ \\
\hline 0 & 0.02 & $0.67 \pm 0.73$ \\
1 & 0.19 & $0.84 \pm 0.89$ \\
2 & 0.06 & $0.80 \pm 1.31$ \\
3 & 0.60 & $0.61 \pm 12.36$ \\
4 & 0.14 & $1.04 \pm 0.67$ \\
5 & 0.77 & $0.67 \pm 0.74$ \\
6 & 2.49 & $1.03 \pm 5.42$ \\
7 & $\ldots$ & $\ldots$ \\
8 & 181.04 & $-0.01 \pm 0.02$ \\
\hline
\end{tabular}

Note. Values are Scaled so to be Compatible with Exponential Fits to The Galactic Profiles $\left(\rho_{0} / 4\right.$ and $\left.z_{0} / 2\right)$.

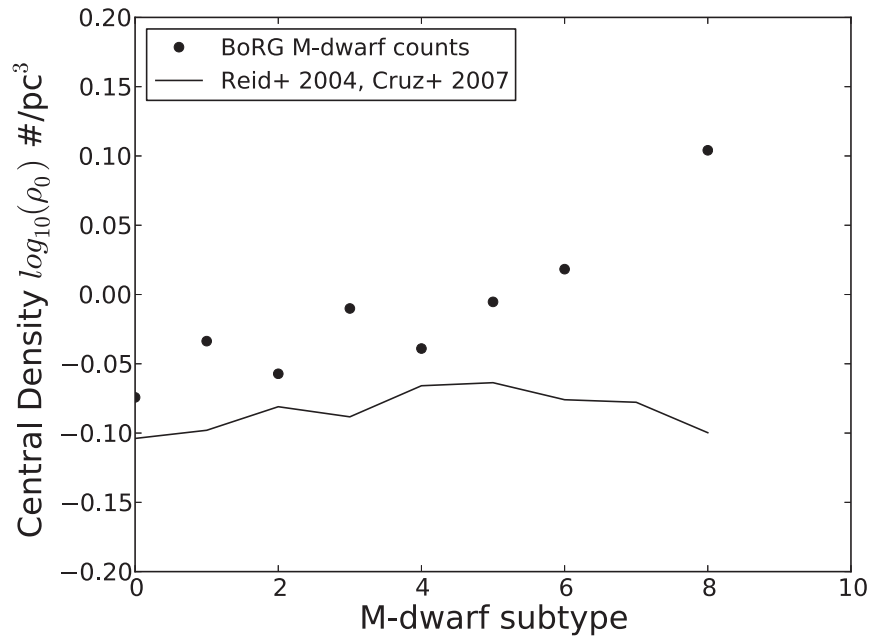

Figure 26. The in-plane central density-the number of M-dwarfs belonging to the disk at the center of the Milky Way-for the best fit to the vertical distribution of M-dwarfs as a function of the M-dwarf subtype. The black circles are the values from Reid et al. (2008) for the immediate Solar neighborhood $(<20 \mathrm{pc})$, renormalized to the center of the Milky Way.

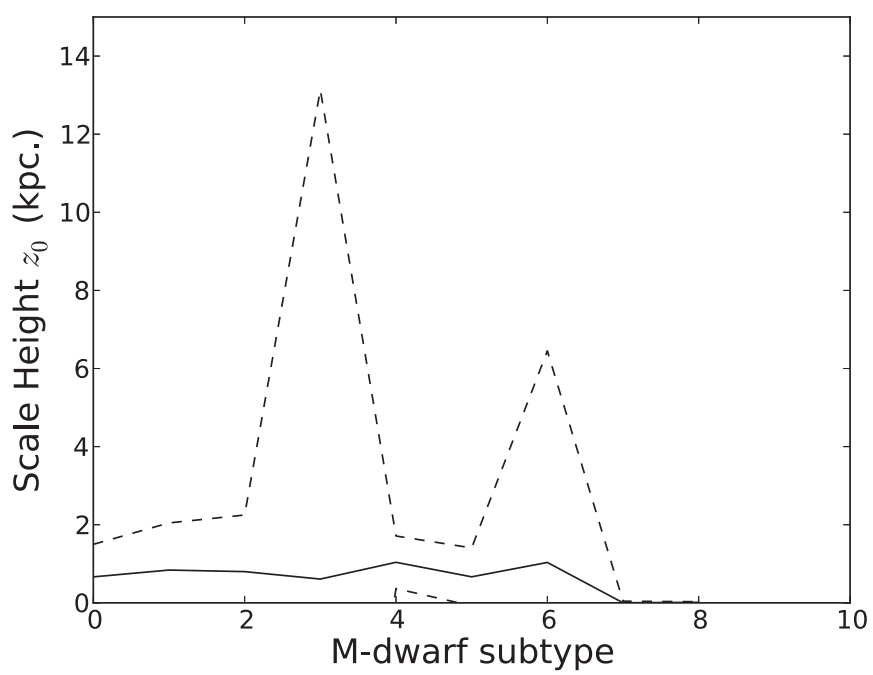

Figure 27. The scale-height $\left(z_{0} / 2\right)$ of the best vertical fit as a function of the M-dwarf subtype. Dashed lines are the uncertainty in the fit. A constant value of $600 \mathrm{pc}$ would be consistent with most of these fits. This is $\sim 2 \times$ greater than expected from previous work. 
Table 14

The 274 M-dwarfs Identified in BoRG

\begin{tabular}{|c|c|c|c|c|c|c|c|c|c|c|c|c|c|c|c|}
\hline $\mathrm{ID}$ & R.A. & Decl. & 1 & b & $m_{F 098 M}$ & $m_{F 125 W}$ & $m_{F 125 W}$ & $m_{F 606 W}$ & Mtype & modulus & dist & Height & Radius & Volume & Area \\
\hline $\begin{array}{l}\text { borg_0110- } \\
\quad 0224.551 .0\end{array}$ & 17.501759 & -2.418265 & 133.918256 & -64.893095 & $23.82 \pm 0.04$ & $23.46 \pm 0.02$ & $23.28 \pm 0.03$ & $25.17 \pm 0.09$ & $2.04 \pm 0.32$ & 16.48 & 19.74 & 14.25 & $16.88 \pm 1.85$ & 228.43 & 10.88 \\
\hline $\begin{array}{l}\text { borg_0110- } \\
\quad 0224.719 .0\end{array}$ & 17.503586 & -2.415663 & 133.921454 & -64.890220 & $24.33 \pm 0.05$ & $23.97 \pm 0.03$ & $23.87 \pm 0.04$ & $25.83 \pm 0.14$ & $2.56 \pm 0.50$ & 16.73 & 22.16 & 15.99 & $17.90 \pm 2.73$ & 287.73 & 10.88 \\
\hline $\begin{array}{l}\text { borg_0110- } \\
\quad 0224.820 .0\end{array}$ & 17.528020 & -2.411389 & 133.976421 & -64.881854 & $19.68 \pm 0.00$ & $19.51 \pm 0.00$ & $19.45 \pm 0.00$ & $22.77 \pm 0.01$ & $7.30 \pm 0.04$ & 8.59 & 0.52 & 0.40 & $8.72 \pm 0.01$ & 0.16 & 10.88 \\
\hline $\begin{array}{l}\text { borg_0110- } \\
\quad 0224.1016 .0\end{array}$ & 17.553100 & -2.408888 & 134.033565 & -64.875102 & $24.54 \pm 0.09$ & $23.90 \pm 0.04$ & $23.72 \pm 0.05$ & $25.39 \pm 0.13$ & $1.30 \pm 0.46$ & 17.18 & 27.26 & 19.63 & $20.08 \pm 3.83$ & 435.66 & 10.88 \\
\hline $\begin{array}{l}\text { borg_0110- } \\
0224.1414 .0\end{array}$ & 17.559120 & -2.400783 & 134.044244 & -64.866085 & $24.08 \pm 0.06$ & $23.71 \pm 0.06$ & $23.44 \pm 0.04$ & $24.40 \pm 0.05$ & $-1.43 \pm 0.27$ & 17.26 & 28.31 & 20.37 & $20.52 \pm 687.31$ & 469.66 & 10.88 \\
\hline
\end{tabular}


Table 15

The $1 \mathrm{~T}$-Dwarfs Identified in BoRG

\begin{tabular}{|c|c|c|c|c|c|c|c|c|}
\hline ID & R.A. & Decl. & 1 & $\mathrm{~b}$ & $m_{F 098 M}$ & $m_{F 125 W}$ & $m_{F 125 W}$ & $m_{F 606 W}$ \\
\hline borg_0540-6409.1929.0 & 84.862502 & -64.152446 & 84.862502 & -64.152446 & $22.9 \pm 0.0$ & $21.9 \pm 0.0$ & $22.2 \pm 0.0$ & $21.5 \pm 0.0$ \\
\hline
\end{tabular}

Table 16

The 30 L-Dwarfs Identified in BoRG

\begin{tabular}{|c|c|c|c|c|c|c|c|c|}
\hline ID & R.A. & Decl. & 1 & $\mathrm{~b}$ & $m_{F 098 M}$ & $m_{F 125 W}$ & $m_{F 125 W}$ & $m_{F 606 W}$ \\
\hline borg_0110-0224.1010.0 & 17.537240 & -2.409470 & 17.537240 & -2.409470 & $24.7 \pm 0.1$ & $23.7 \pm 0.0$ & $23.5 \pm 0.0$ & $25.3 \pm 0.1$ \\
\hline borg_0439-5317.882.0 & 69.836642 & -53.263207 & 69.836642 & -53.263207 & $24.0 \pm 0.0$ & $22.7 \pm 0.0$ & $22.2 \pm 0.0$ & $24.8 \pm 0.9$ \\
\hline borg_0540-6409.2993.0 & 84.876906 & -64.132531 & 84.876906 & -64.132531 & $25.0 \pm 0.1$ & $23.8 \pm 0.0$ & $23.4 \pm 0.0$ & $26.8 \pm 0.6$ \\
\hline borg_0808+3946.457.0 & 122.094091 & 39.759095 & 122.094091 & 39.759095 & $25.0 \pm 0.1$ & $23.9 \pm 0.0$ & $23.5 \pm 0.0$ & $26.9 \pm 0.6$ \\
\hline borg_0819+4911.264.0 & 124.831743 & 49.175806 & 124.831743 & 49.175806 & $23.9 \pm 0.0$ & $22.8 \pm 0.0$ & $22.4 \pm 0.0$ & $27.9 \pm 1.6$ \\
\hline borg_0906+0255.462.0 & 136.409407 & 2.923645 & 136.409407 & 2.923645 & $24.4 \pm 0.0$ & $23.3 \pm 0.0$ & $22.8 \pm 0.0$ & $27.6 \pm 0.8$ \\
\hline borg_0926+4426.581.0 & 141.355847 & 44.425445 & 141.355847 & 44.425445 & $24.3 \pm 0.0$ & $23.1 \pm 0.0$ & $22.6 \pm 0.0$ & $25.2 \pm 0.9$ \\
\hline borg_1103-2330.623.0 & 165.819910 & -23.506010 & 165.819910 & -23.506010 & $23.9 \pm 0.1$ & $22.9 \pm 0.0$ & $22.5 \pm 0.0$ & $25.7 \pm 0.2$ \\
\hline borg_1111+5545.1125.0 & 167.747157 & 55.771759 & 167.747157 & 55.771759 & $23.6 \pm 0.0$ & $22.5 \pm 0.0$ & $22.1 \pm 0.0$ & $26.6 \pm 0.3$ \\
\hline borg_1153+0056.247.0 & 178.200215 & 0.922794 & 178.200215 & 0.922794 & $23.9 \pm 0.0$ & $22.9 \pm 0.0$ & $22.4 \pm 0.0$ & $26.2 \pm 0.3$ \\
\hline borg_1230+0750.2419.0 & 187.471709 & 7.820117 & 187.471709 & 7.820117 & $23.9 \pm 0.1$ & $22.8 \pm 0.0$ & $22.3 \pm 0.0$ & $26.2 \pm 0.3$ \\
\hline borg_1301+0000.1067.0 & 195.326828 & 0.013297 & 195.326828 & 0.013297 & $23.5 \pm 0.1$ & $22.2 \pm 0.0$ & $21.8 \pm 0.0$ & $24.3 \pm 0.9$ \\
\hline borg_1632+3733.1325.0 & 248.076265 & 37.572819 & 248.076265 & 37.572819 & $24.3 \pm 0.0$ & $23.0 \pm 0.1$ & $22.5 \pm 0.0$ & $26.7 \pm 0.5$ \\
\hline borg_2132+1004.284.0 & 323.058751 & 10.068991 & 323.058751 & 10.068991 & $16.8 \pm 0.0$ & $15.0 \pm 0.0$ & $14.7 \pm 0.0$ & $17.2 \pm 0.9$ \\
\hline borg_2132+1004.332.0 & 323.060235 & 10.059801 & 323.060235 & 10.059801 & $21.2 \pm 0.0$ & $19.9 \pm 0.0$ & $19.7 \pm 0.0$ & $22.2 \pm 0.9$ \\
\hline borg_2155-4411.227.0 & 328.831076 & -44.185153 & 328.831076 & -44.185153 & $23.5 \pm 0.0$ & $22.5 \pm 0.0$ & $22.2 \pm 0.0$ & $24.8 \pm 0.9$ \\
\hline borg_2155-4411.974.0 & 328.809893 & -44.164189 & 328.809893 & -44.164189 & $24.3 \pm 0.0$ & $23.0 \pm 0.0$ & $22.5 \pm 0.0$ & $25.1 \pm 0.9$ \\
\hline borg_2351-4332.1314.0 & 357.668728 & -43.514053 & 357.668728 & -43.514053 & $25.2 \pm 0.1$ & $23.9 \pm 0.0$ & $23.5 \pm 0.0$ & $26.0 \pm 0.9$ \\
\hline borg_0456-2203.920.0 & 73.945566 & -22.049396 & 73.945566 & -22.049396 & $24.6 \pm 0.1$ & $23.4 \pm 0.0$ & $23.2 \pm 0.0$ & $24.4 \pm 0.0$ \\
\hline borg_1059+0519.644.0 & 164.717943 & 5.301323 & 164.717943 & 5.301323 & $24.4 \pm 0.0$ & $23.1 \pm 0.0$ & $22.6 \pm 0.0$ & $26.5 \pm 0.3$ \\
\hline borg_1118-1858.1915.0 & 169.392755 & -18.972497 & 169.392755 & -18.972497 & $24.8 \pm 0.0$ & $23.8 \pm 0.0$ & $23.3 \pm 0.0$ & $26.1 \pm 0.1$ \\
\hline borg_1459+7146.1298.0 & 224.751450 & 71.780685 & 224.751450 & 71.780685 & $24.8 \pm 0.2$ & $23.5 \pm 0.0$ & $23.1 \pm 0.0$ & $25.7 \pm 0.1$ \\
\hline borg_2132-1202.6461.0 & 322.941469 & -12.021619 & 322.941469 & -12.021619 & $24.6 \pm 0.1$ & $23.4 \pm 0.0$ & $23.0 \pm 0.0$ & $26.5 \pm 0.4$ \\
\hline
\end{tabular}

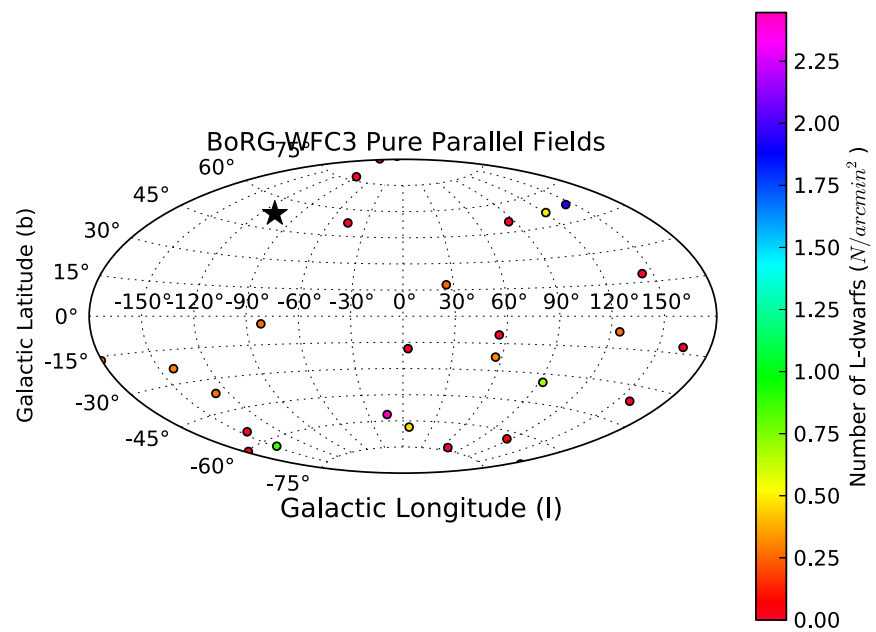

Figure 28. The distribution of BoRG fields with the number of L-dwarfs indicated. Other symbols identical to Figure 22. 
component well above the plane of the disk, i.e., we are including the density of Halo stars in our disk fit. A 0.1 dex offset suggest of order $10 \%$ of the stars in are, in fact, Halo stars (see also van Vledder et al. 2016).

The values in Figure 27 and Table 10 are still too high to be consistent with previous work on the vertical scale of stars in the thin disk. The vertical $\operatorname{sech}^{2}$ profile does not describe the density distribution well above $\sim 500 \mathrm{pc}$ and this new distributions highlights the need to include another component well above and in addition to the disk.

For completeness, we include Figure 28 which shows the surface density of L-dwarfs selected by our morphology and color criteria.

\section{CONCLUSIONS}

The majority of conclusions for Holwerda et al. (2014) remain the same, only the last two change:

1. The secondary component visible in the vertical distribution of M-dwarfs is likely the Halo and not the thick disk of the Milky Way (Figures 24 and 25).

2. A naive, single-component fit of the vertical distribution of M-dwarfs shows no dependence on M-dwarf subtype and a scaleheight for all M-dwarf subtypes that is still too high to be consistent with previous measures of the scale-height: $z_{0} \sim 600 \mathrm{pc}$, (Figure 27), a result of the second structural component.

The authors would like to thank the referee, K. Cruz for her help with the erratum. This research made use of Astropy, a community-developed core Python package for Astronomy (Astropy Collaboration et al. 2013). This research made use of matplotlib, a Python library for publication quality graphics (Hunter 2007). PyRAF is a product of the Space Telescope Science Institute, which is operated by AURA for NASA. This research made use of SciPy (Jones et al. 2001).

\section{REFERENCES}

Astropy Collaboration, Robitaille, T. P., Tollerud, E. J., et al. 2013, A\&A, 558, A33

Comerón, S., Elmegreen, B. G., Knapen, J. H., et al. 2011b, ApJ, 738, L17

Comerón, S., Elmegreen, B. G., Knapen, J. H., et al. 2011c, ApJ, 741, 28

Comerón, S., Knapen, J. H., Sheth, K., et al. 2011c, ApJ, 729, 18

Cruz, K. L., Reid, I. N., Kirkpatrick, J. D., et al. 2007, AJ, 133, 439

Hawley, S. L., Covey, K. R., Knapp, G. R., et al. 2002, AJ, 123, 3409

Holwerda, B. W., Trenti, M., Clarkson, W., et al. 2014, ApJ, 788, 77
Hunter, J. D. 2007, Computing In Science \& Engineering, 9, 90

Jones, E., Oliphant, T., Peterson, P., et al. 2001, SciPy: Open source scientific tools for Python

Reid, I. N., Cruz, K. L., Allen, P., et al. 2004, AJ, 128, 463

Reid, I. N., Cruz, K. L., Kirkpatrick, J. D., et al. 2008, AJ, 136, 1290

Ryan, R. E., Jr., \& Reid, I. N. 2016, AJ, 151, 92

van Vledder, Is., van der Vlugt, D., Holwerda, B. W., Kenworthy, M. A. Bouwens, R. J., \& Trenti, M. 2016, MNRAS, 458, 425 